\title{
Operator theory: quantum white noise approach
}

\author{
Abdessatar Barhoumi • Bilel Kacem Ben Ammou • \\ Hafedh Rguigui
}

Received: 28 November 2014 / Accepted: 5 February 2015 / Published online: 21 February 2015

(C) Chapman University 2015

\begin{abstract}
we develop an operator theory on a nuclear algebra of white noise operators in terms of the quantum white noise (QWN) derivatives and their dual adjoints. Using an adequate definition of a QWN-symbol transformation, we discuss QWN-integral-sum kernel operators which give the Fock expansion of the QWN-operators (i.e. the linear operators acting on nuclear algebra of white noise operators). As application, we characterize all rotation invariant QWN-operators by means of the QWN-conservation operator, the QWN-Gross Laplacians. These topics are expected to open a new area in QWN infinite-dimensional analysis.
\end{abstract}

Keywords QWN-derivatives · QWN-operators · QWN-symbol map · Rotation-invariant QWN-operators · QWN-Gross Laplacian $\cdot$ QWN-conservation operator

Mathematics Subject Classification Primary 60H40; Secondary 46A32, 46F25, 46G20

\section{Introduction}

The white noise analysis has been developed to an infinite-dimensional distribution theory on Gaussian space $\left(E^{\prime}, \mu\right)$ as an infinite-dimensional analogue of Schwartz distribution theory on Euclidean space $\mathbb{R}$ with Lebesgue measure:

$E:=\mathscr{S}(\mathbb{R}) \subset H:=L^{2}(\mathbb{R}, d x) \subset \mathscr{S}^{\prime}(\mathbb{R})=: E^{\prime}$.

\footnotetext{
A. Barhoumi - B. K. B. Ammou Department of Mathematics, Nabeul Preparatory Engineering Institute, Carthage University, Campus Universitaire, Mrezgua, 8000 Nabeul, Tunisia

e-mail: abdessatar.barhoumi@ipein.rnu.tn

B. K. B. Ammou

e-mail: bilelbenammou@gmail.com

H. Rguigui ( $\square)$

Department of Mathematics, Higher School of Sciences and Technologies of Hammam-Sousse,

Sousse University, Sousse, Tunisia

e-mail: hafedh.rguigui@yahoo.fr
} 
The mathematical framework of white noise analysis is the Gel'fand triple of test function space $\mathscr{F}_{\theta}\left(N^{\prime}\right)$ and generalized function space $\mathscr{F}_{\theta}^{*}\left(N^{\prime}\right)$ :

$\mathscr{F}_{\theta}\left(N^{\prime}\right) \subset L^{2}\left(E^{\prime}, \mu\right) \subset \mathscr{F}_{\theta}^{*}\left(N^{\prime}\right)$.

There has been observed formal analogy between white noise calculus and the calculus on Euclidean space based on this Gel'fand triple, e.g., rotation groups [14], Laplacians [8,13]. The main tools of investigation in the abovementioned papers are the symbol (or Wick symbol) transform of an operator, the Fock expansion and integral kernels operators.

In white noise analysis, the set $\{x(t) ; t \in \mathbb{R}\}$ is taken as a coordinate system of $\left(E^{\prime}, \mu\right)$ and $\left\{a_{t}, a_{t}^{*} ; t \in \mathbb{R}\right\}$ (annihilation and creation) is the coordinate system for white noise differential operators as homologue of the

Euclidean differential basis $\left\{\frac{\partial}{\partial x_{k}} ; 1 \leq k \leq \mathrm{d}\right\}$. It is a fundamental fact that every white noise operator $\Xi \in$ $\mathscr{L}\left(\mathscr{F}_{\theta}\left(N^{\prime}\right), \mathscr{F}_{\theta}^{*}\left(N^{\prime}\right)\right)$ admits a Fock expansion as an infinite series:

$\Xi=\sum_{l, m=0}^{\infty} \Xi_{l, m}\left(\kappa_{l, m}\right)$,

where the integral kernel operator $\Xi_{l, m}\left(\kappa_{l, m}\right)$ is expressed in a formal integral

$\Xi_{l, m}\left(\kappa_{l, m}\right)=\int_{\mathbb{R}^{l+m}} \kappa_{l, m}\left(s_{1}, \cdots, s_{l}, t_{1}, \cdots, t_{m}\right) a_{s_{1}}^{*} \cdots a_{s_{l}}^{*} a_{t_{1}} \cdots a_{t_{m}} \mathrm{~d} s_{1} \cdots \mathrm{d} s_{l} \mathrm{~d} t_{1} \cdots \mathrm{d} t_{m}$.

Accordingly, the white noise operator $\Xi$ can be regarded as a "function" of the variables $\left\{a_{s}, a_{t}^{*} ; s, t \in \mathbb{R}\right\}$. This intuitive idea motivated Ji-Obata (see Ref. [16]) to introduce the so-called quantum white noise derivatives

$D_{t}^{+} \Xi=\frac{\partial \Xi}{\partial a_{t}} \equiv\left[a_{t}, \Xi\right], \quad D_{t}^{-} \Xi=\frac{\partial \Xi}{\partial a_{t}^{*}} \equiv-\left[a_{t}^{*}, \Xi\right]$

acting on a suitable subset of the nuclear algebra $\mathscr{L}\left(\mathscr{F}_{\theta}\left(N^{\prime}\right), \mathscr{F}_{\theta}^{*}\left(N^{\prime}\right)\right)$. The set

$\left\{D_{s}^{+}, D_{t}^{-},\left(D_{u}^{+}\right)^{*},\left(D_{v}^{-}\right)^{*} ; s, t, u, v \in \mathbb{R}\right\}$

will be taken as a quantum white noise coordinate system.

The main purpose of this paper is to develop operator theory on a nuclear algebra of white noise operators; we give the Fock expansion of the QWN-operators in terms of a QWN-integral kernel operators which are defined by the QWN-symbol map and expressed in terms of the quantum white noise coordinate system. The above Fock expansion will play a key role in our discussion, in particular using the quantum white noise analogues of the Gross Laplacian and the number operator, we characterize the rotation-invariant QWN operators.

The paper is organized as follows. In Sect. 2, we summarize the common notations, concepts and basic topological isomorphisms used throughout the paper. In Sect. 3, we introduce the QWN-integral kernel operator using the quantum white noise coordinate system. In Sect. 4, we define the QWN-symbol map and study its properties. In Sect. 5, the chaotic expansion of the QWN-operators is given in terms of the QWN coordinate system. In Sect. 6, we characterize all rotation-invariant QWN-operators.

\section{Preliminaries}

In this section, we summarize the common notations and concepts used throughout the paper which can be found in Refs. [5-7, 10, 19, 21, 23, 24, 28,29].

\subsection{Basic Gel'fand triples}

Let $H$ be the real Hilbert space of square integrable functions on $\mathbb{R}$ with norm $|\cdot|_{0}$. The Gel'fand triple (1) can be reconstructed in a standard way (see Ref. [21]) by the harmonic oscillator $A=1+t^{2}-d^{2} / d t^{2}$ and $H$. The 
eigenvalues of $A$ are $2 n+2, n=0,1,2, \cdots$ and the corresponding eigenfunctions $\left\{e_{n} ; n \geq 0\right\}$ form an orthonormal basis for $L^{2}(\mathbb{R})$. In fact, $\left(e_{n}\right)$ are the Hermite functions and therefore each $e_{n}$ is an element of $E$. The space $E$ is a nuclear space equipped with the Hilbertian norms

$|\xi|_{p}=\left|A^{p} \xi\right|_{0}, \quad \xi \in E, \quad p \in \mathbb{R}$

and we have

$E=\operatorname{proj} \lim _{p \rightarrow \infty} E_{p}, \quad E^{\prime}=$ ind $\lim _{p \rightarrow \infty} E_{-p}$,

where for $p \geq 0, E_{p}$ is the completion of $E$ with respect to the norm $|\cdot|_{p}$ and $E_{-p}$ is the topological dual space of $E_{p}$. We denote by $N=E+i E$ and $N_{p}=E_{p}+i E_{p}, p \in \mathbb{Z}$, the complexifications of $E$ and $E_{p}$, respectively.

Throughout the paper, we fix a Young function $\theta$ that satisfies the following condition

$\limsup _{x \rightarrow \infty} \frac{\theta(x)}{x^{2}}<\infty$.

The polar function $\theta^{*}$ of $\theta$, defined by $\theta^{*}(x)=\sup _{t \geq 0}(t x-\theta(t)), x \geq 0$, is also a Young function. For more details, see Refs. [10,23]. For a complex Banach space $(B,\|\cdot\|)$, let $\mathscr{H}(B)$ denotes the space of all entire functions on $B$. For each $m>0$ we denote by $\operatorname{Exp}(B, \theta, m)$ to be

$\operatorname{Exp}(B, \theta, m)=\left\{f \in \mathscr{H}(B) ; \quad\|f\|_{\theta, m}:=\sup _{z \in B}|f(z)| e^{-\theta(m\|z\|)}<\infty\right\}$.

The two spaces $\mathscr{F}_{\theta}\left(N^{\prime}\right)$ and $\mathscr{G}_{\theta}(N)$ are defined by

$\mathscr{F}_{\theta}\left(N^{\prime}\right)=\operatorname{projlim}_{p \rightarrow \infty ; m \downarrow 0} \operatorname{Exp}\left(N_{-p}, \theta, m\right), \quad \mathscr{G}_{\theta}(N)=\operatorname{ind}_{p \rightarrow \infty ; m \downarrow 0} \operatorname{Exp}\left(N_{p}, \theta, m\right)$.

In the remainder of this paper, we simply use $\mathscr{F}_{\theta}$ for $\mathscr{F}_{\theta}\left(N^{\prime}\right)$. It is noteworthy that, for each $\xi \in N$, the exponential function $e_{\xi}(z):=e^{\langle z, \xi\rangle}, \quad z \in N^{\prime}$, belongs to $\mathscr{F}_{\theta}$ and the set of such test functions spans a dense subspace of $\mathscr{F}_{\theta}$. The space of linear continuous operators from $\mathscr{F}_{\theta}$ into its topological dual space $\mathscr{F}_{\theta}^{*}$ is denoted by $\mathscr{L}\left(\mathscr{F}_{\theta}, \mathscr{F}_{\theta}^{*}\right)$ and assumed to carry the bounded convergence topology. Let $\mu$ be the standard Gaussian measure on $E^{\prime}$ uniquely specified by its characteristic function

$e^{-\frac{1}{2}|\xi|_{0}^{2}}=\int_{E^{\prime}} e^{i\langle x, \xi\rangle} \mu(\mathrm{d} x), \quad \xi \in E$.

Under condition (5), we have the nuclear Gel'fand triple (2), see Ref. [10].

\subsection{QWN-derivatives}

For $z \in N^{\prime}$ and $\varphi(x)$ with Taylor expansion $\sum_{n=0}^{\infty}\left\langle x^{\otimes n}, f_{n}\right\rangle$ in $\mathscr{F}_{\theta}$, the holomorphic derivative of $\varphi$ at $x \in N^{\prime}$ in the direction $z$ is defined by $(a(z) \varphi)(x):=\lim _{\lambda \rightarrow 0} \frac{\varphi(x+\lambda z)-\varphi(x)}{\lambda}$. We can check that the limit always exists, $a(z) \in \mathscr{L}\left(\mathscr{F}_{\theta}, \mathscr{F}_{\theta}\right)$ and $a^{*}(z) \in \mathscr{L}\left(\mathscr{F}_{\theta}^{*}, \mathscr{F}_{\theta}^{*}\right)$, where $a^{*}(z)$ is the adjoint of $a(z)$. For $\zeta \in N, a(\zeta)$ extends to a continuous linear operator from $\mathscr{F}_{\theta}^{*}$ into itself (denoted by the same symbol) and $a^{*}(\zeta)$ (restricted to $\mathscr{F}_{\theta}$ ) is a continuous linear operator from $\mathscr{F}_{\theta}$ into itself. If $z=\delta_{t} \in E^{\prime}$ we simply write $a_{t}$ instead of $a\left(\delta_{t}\right)$. In QWN-field theory $a_{t}$ and $a_{t}^{*}$ are called the annihilation and creation operators at the point $t \in \mathbb{R}$.

The symbol and the Wick symbol, denoted by $\sigma$ and $\omega$ respectively, of $\Xi \in \mathscr{L}\left(\mathscr{F}_{\theta}, \mathscr{F}_{\theta}^{*}\right)$ are by definition ([21]) the $\mathbb{C}$-valued function on $N \times N$ obtained as

$\sigma(\Xi)(\xi, \eta)=\left\langle\left\langle\Xi e_{\xi}, e_{\eta}\right\rangle, \quad \omega(\Xi)(\xi, \eta)=\left\langle\left\langle\Xi e_{\xi}, e_{\eta}\right\rangle\right\rangle e^{-\langle\xi, \eta\rangle}, \quad \xi, \eta \in N\right.$,

respectively, where $\langle\langle\cdot, \cdot\rangle\rangle$ denotes the duality between the two spaces $\mathscr{F}_{\theta}^{*}$ and $\mathscr{F}_{\theta}$.

It is a fundamental fact in quantum white noise theory [21] (see also Ref. [17]) that every white noise operator $\Xi \in \mathscr{L}\left(\mathscr{F}_{\theta}, \mathscr{F}_{\theta}^{*}\right)$ admits a unique Fock expansion (3) where, for each pairing $l, m \geq 0, \kappa_{l, m} \in\left(N^{\otimes(l+m)}\right)_{s y m(l, m)}^{\prime}$ and $\Xi_{l, m}\left(\kappa_{l, m}\right)$ is the integral kernel operator characterized via the Wick symbol transform by

$\omega\left(\Xi_{l, m}\left(\kappa_{l, m}\right)\right)(\xi, \eta)=\left\langle\kappa_{l, m}, \eta^{\otimes l} \otimes \xi^{\otimes m}\right\rangle, \quad \xi, \eta \in N$. 
This can be formally rewritten as (4). For $\zeta \in N$, the quantum white noise derivatives are defined by

$D_{\zeta}^{+} \Xi=[a(\zeta), \Xi], \quad D_{\zeta}^{-} \Xi=-\left[a^{*}(\zeta), \Xi\right]$.

These are called the creation derivative and annihilation derivative of $\Xi$, respectively. In Ref. [1], for $z \in N^{\prime}$, it is shown that $D_{z}^{+}$is a continuous operator from $\mathscr{L}\left(\mathscr{F}_{\theta}, \mathscr{F}_{\theta}\right)$ into itself and $D_{z}^{-}$is a continuous operator from $\mathscr{L}\left(\mathscr{F}_{\theta}^{*}, \mathscr{F}_{\theta}^{*}\right)$ into itself. The pointwisely quantum white noise derivatives $D_{t}^{ \pm} \equiv D_{\delta_{t}}^{ \pm}$are discussed in Ref. [16].

\subsection{Basic topological isomorphisms}

Let $\mathscr{G}_{\theta^{*}}(N \oplus N)$ denotes the nuclear space obtained as in (6) by replacing $N_{p}$ with $N_{p} \oplus N_{p}$.

Theorem 1 (See Ref. [17]) The symbol and the Wick symbol maps realize two topological isomorphisms between $\mathscr{L}\left(\mathscr{F}_{\theta}, \mathscr{F}_{\theta}^{*}\right)$ and $\mathscr{G}_{\theta^{*}}(N \oplus N)$.

For $p \in \mathbb{N}$ and $\gamma_{1}, \gamma_{2}>0$, we define the Hilbert spaces

$F_{\theta, \gamma_{1}, \gamma_{2}}\left(N_{p} \oplus N_{p}\right)=\left\{\vec{\varphi}=\left(\varphi_{l, m}\right)_{l, m=0}^{\infty} ; \varphi_{l, m} \in\left(N_{p}^{\otimes l} \otimes N_{p}^{\otimes m}\right)_{s y m(l, m)},\|\vec{\varphi}\|_{\theta, p,\left(\gamma_{1}, \gamma_{2}\right)}^{2}<\infty\right\}$,

where $\|\vec{\varphi}\|_{\theta, p,\left(\gamma_{1}, \gamma_{2}\right)}^{2}:=\sum_{l, m=0}^{\infty}\left(\theta_{l} \theta_{m}\right)^{-2} \gamma_{1}^{-l} \gamma_{2}^{-m}\left|\varphi_{l, m}\right|_{p}^{2}$ and $\theta_{n}=\inf _{r>0} e^{\theta(r)} / r^{n}, n \in \mathbb{N}$. Put $F_{\theta}(N \oplus N)=$ $\bigcap_{p \in \mathbb{N}, \gamma_{1}, \gamma_{2}>0} F_{\theta, \gamma_{1}, \gamma_{2}}\left(N_{p} \oplus N_{p}\right)$. Let $\mathscr{H}_{\theta}(N \oplus N)=\bigcap_{p \geq 0, \gamma_{1}, \gamma_{2}>0} \operatorname{Exp}\left(N_{p} \oplus N_{p}, \theta, \gamma_{1}, \gamma_{2}\right)$, where $\operatorname{Exp}\left(N_{p} \oplus\right.$ $\left.N_{p}, \theta, \gamma_{1}, \gamma_{2}\right)$ denotes the space of all entire functions on $N_{p} \times N_{p}$ such that

$\sup _{\left(x_{1}, x_{2}\right) \in\left(N_{p} \times N_{p}\right)}\left|g\left(x_{1}, x_{2}\right)\right| e^{-\theta\left(\gamma_{1}\left|x_{1}\right| p\right)-\theta\left(\gamma_{2}\left|x_{2}\right| p\right)}<\infty$.

In other words, from the topological isomorphism between $\mathscr{H}_{\theta}(N \oplus N)$ and $F_{\theta}(N \oplus N)$ which can be easily shown (see [10,17]), all holomorphic functions $g$ in $\mathscr{H}_{\theta}(N \oplus N)$ admit a Taylor expansion $g\left(x_{1}, x_{2}\right)=\sum_{l, m}\left\langle g_{l, m}, x_{2}^{\otimes l} \otimes\right.$ $\left.x_{1}^{\otimes m}\right\rangle$ for $x_{1}, x_{2} \in N$, where $g_{l, m} \in\left(N^{\otimes(l+m)}\right)_{\operatorname{sym}(l, m)}$ is such that $\|\vec{g}\|_{\theta, p,\left(\gamma_{1}, \gamma_{2}\right)}^{2}<\infty$ for all $p \in \mathbb{N}$ and $\gamma_{1}$, $\gamma_{2}>0$.

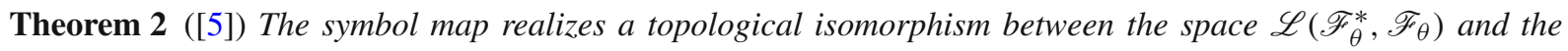
space $\mathscr{H}_{\theta}(N \oplus N)$.

For any $S_{1}, S_{2} \in \mathscr{L}\left(\mathscr{F}_{\theta}, \mathscr{F}_{\theta}^{*}\right)$, the the Wick product of $S_{1}$ and $S_{2}$ is defined by

$\omega\left(S_{1} \diamond S_{2}\right)=\omega\left(S_{1}\right) \omega\left(S_{2}\right)$.

Theorem 3 ([5]) An operator $\Xi \in \mathscr{L}\left(\mathscr{F}_{\theta}^{*}, \mathscr{F}_{\theta}\right)$ iff there exists a unique $\left(\kappa_{l, m}\right)_{l, m} \in F_{\theta}(N \oplus N)$ such that

$$
\Xi=\Xi_{-\tau} \diamond \sum_{l, m=0}^{\infty} \Xi_{l, m}\left(\kappa_{l, m}\right)
$$

where $\Xi_{-\tau}$ is given by $\Xi_{-\tau}=\sum_{k=0}^{\infty} \frac{(-1)^{k}}{k !} \Xi_{k, k}\left(\tau^{\otimes k}\right)$.

Let $\mathscr{U}_{\theta}=\omega^{-1}\left(\mathscr{H}_{\theta}(N \oplus N)\right)$. By a simple computation one can show that $\Xi \in \mathscr{U}_{\theta}$ iff there exists a unique $\left(\kappa_{l, m}\right)_{l, m=0}^{\infty} \in F_{\theta}(N \oplus N)$ such that $\Xi=\sum_{l, m=0}^{\infty} \Xi_{l, m}\left(\kappa_{l, m}\right)$, i.e.,

$\mathscr{U}_{\theta}=\left\{\Xi=\sum_{l, m=0}^{\infty} \Xi_{l, m}\left(\kappa_{l, m}\right),\left(\kappa_{l, m}\right)_{l, m=0}^{\infty} \in F_{\theta}(N \oplus N)\right\}$.

Theorem 4 ([5]) The Wick symbol map realizes a topological isomorphism between the space $\mathscr{U}_{\theta}$ and the space $\mathscr{H}_{\theta}(N \oplus N)$. 
From Theorems 1 and 2, we have the topological isomorphism:

$$
\begin{aligned}
& \mathscr{L}\left(\mathscr{F}_{\theta}, \mathscr{F}_{\theta}^{*}\right) \simeq \mathscr{G}_{\theta^{*}}(N \oplus N)=\bigcup_{p \geq 0, \gamma_{1}, \gamma_{2}>0} \operatorname{Exp}\left(N_{p} \oplus N_{p}, \theta^{*},\left(\gamma_{1}, \gamma_{2}\right)\right) \\
& \mathscr{L}\left(\mathscr{F}_{\theta}^{*}, \mathscr{F}_{\theta}\right) \simeq \mathscr{H}_{\theta}(N \oplus N)=\bigcap_{p \geq 0, \gamma_{1}, \gamma_{2}>0} \operatorname{Exp}\left(N_{p} \oplus N_{p}, \theta,\left(\gamma_{1}, \gamma_{2}\right)\right) .
\end{aligned}
$$

For $p \geq 0$ and $\gamma_{1}, \gamma_{2}>0$, let $\mathscr{L}_{\theta,-p,\left(\gamma_{1}, \gamma_{2}\right)}\left(\mathscr{F}_{\theta}, \mathscr{F}_{\theta}^{*}\right)$ denotes the subspace of all $\Xi \in \mathscr{L}\left(\mathscr{F}_{\theta}, \mathscr{F}_{\theta}^{*}\right)$ which correspond to elements in $\operatorname{Exp}\left(N_{p} \oplus N_{p}, \theta^{*},\left(\gamma_{1}, \gamma_{2}\right)\right)$. Similarly, let $\mathscr{L}_{\theta, p,\left(\gamma_{1}, \gamma_{2}\right)}\left(\mathscr{F}_{\theta}^{*}, \mathscr{F}_{\theta}\right)$ denotes the subspace of all $\Xi \in \mathscr{L}\left(\mathscr{F}_{\theta}^{*}, \mathscr{F}_{\theta}\right)$ which correspond to elements in $\operatorname{Exp}\left(N_{p} \oplus N_{p}, \theta,\left(\gamma_{1}, \gamma_{2}\right)\right)$. The topology of $\mathscr{L}_{\theta,-p,\left(\gamma_{1}, \gamma_{2}\right)}\left(\mathscr{F}_{\theta}, \mathscr{F}_{\theta}^{*}\right)$ is naturally induced from the norm of the Banach space $\operatorname{Exp}\left(N_{p} \oplus N_{p}, \theta^{*},\left(\gamma_{1}, \gamma_{2}\right)\right)$ which will be denoted by $\|\cdot\|_{\theta,-p,\left(\gamma_{1}, \gamma_{2}\right)}$, i.e., for $\Xi \in \mathscr{L}_{\theta,-p,\left(\gamma_{1}, \gamma_{2}\right)}\left(\mathscr{F}_{\theta}, \mathscr{F}_{\theta}^{*}\right)$,

$\|\Xi\|_{\theta,-p,\left(\gamma_{1}, \gamma_{2}\right)}=\|\omega(\Xi)\|_{\theta^{*},-p,\left(\gamma_{1}, \gamma_{2}\right)}=\sup _{\xi, \eta \in N_{p}}|\omega(\Xi)(\xi, \eta)| e^{-\theta^{*}\left(\gamma_{1}|\xi|_{p}\right)-\theta^{*}\left(\gamma_{2}|\eta|_{p}\right)}$.

Similarly, the topology of $\mathscr{L}_{\theta, p,\left(\gamma_{1}, \gamma_{2}\right)}\left(\mathscr{F}_{\theta}^{*}, \mathscr{F}_{\theta}\right)$ is naturally induced from the norm of the Banach $\operatorname{space} \operatorname{Exp}\left(N_{p} \oplus\right.$ $\left.N_{p}, \theta,\left(\gamma_{1}, \gamma_{2}\right)\right)$ which will be denoted by $\|\cdot\|_{\theta, p,\left(\gamma_{1}, \gamma_{2}\right)}$, i.e., for $\Xi \in \mathscr{L}_{\theta, p,\left(\gamma_{1}, \gamma_{2}\right)}\left(\mathscr{F}_{\theta}^{*}, \mathscr{F}_{\theta}\right)$ and for all $p \geq 0$ and $\gamma_{1}, \gamma_{2}>0$

$\|\Xi\|_{\theta, p,\left(\gamma_{1}, \gamma_{2}\right)}=\|\sigma(\Xi)\|_{\theta, p,\left(\gamma_{1}, \gamma_{2}\right)}=\sup _{\xi, \eta \in N_{p}}|\sigma(\Xi)(\xi, \eta)| e^{-\theta\left(\gamma_{1}|\xi|_{p}\right)-\theta\left(\gamma_{2}|\eta|_{p}\right)}$.

Via Theorem 4 , the topology of $\mathscr{U}_{\theta}$ is governed by the family of seminorms

$\|\Xi\|_{\theta, p,\left(\gamma_{1}, \gamma_{2}\right)}=\|\omega(\Xi)\|_{\theta, p,\left(\gamma_{1}, \gamma_{2}\right)}=\sup _{\xi, \eta \in N_{p}}|\omega(\Xi)(\xi, \eta)| e^{-\theta\left(\gamma_{1}|\xi|_{p}\right)-\theta\left(\gamma_{2}|\eta|_{p}\right)}$.

Theorem 5 ([5]) The map $f_{\tau}$ defined by $f_{\tau}: \mathscr{L}\left(\mathscr{F}_{\theta}^{*}, \mathscr{F}_{\theta}\right) \longrightarrow \mathscr{U}_{\theta}, \quad \Xi \longmapsto \Xi_{\tau} \diamond \Xi$, is an isometry topological isomorphism.

Recall that, for $p \in \mathbb{N}$ and $\gamma_{1}, \gamma_{2}>0$, we define the Hilbert space

$G_{\theta, \gamma_{1}, \gamma_{2}}\left(N_{-p} \oplus N_{-p}\right)=\left\{\vec{\Phi}=\left(\Phi_{l, m}\right)_{l, m=0}^{\infty} ; \Phi_{l, m} \in N_{-p}^{\widehat{\otimes}(l+m)},\|\vec{\Phi}\|_{\theta,-p,\left(\gamma_{1}, \gamma_{2}\right)}^{2}<\infty\right\}$

where

$\|\vec{\Phi}\|_{\theta,-p,\left(\gamma_{1}, \gamma_{2}\right)}^{2}=\sum_{l, m=0}^{\infty}\left(l ! m ! \theta_{l} \theta_{m}\right)^{2} \gamma_{1}^{l} \gamma_{2}^{m}\left|\Phi_{l, m}\right|_{-p}^{2}$.

Put $G_{\theta}\left(N^{\prime} \oplus N^{\prime}\right)=\bigcup_{p \in \mathbb{N}, \gamma_{1}, \gamma_{2}>0} G_{\theta, \gamma_{1}, \gamma_{2}}\left(N_{-p} \oplus N_{-p}\right)$. The space $G_{\theta}\left(N^{\prime} \oplus N^{\prime}\right)$ carries the dual topology of $F_{\theta}(N \oplus N)$ with respect to the $\mathbb{C}$-bilinear pairing given by $\langle\langle\vec{\Phi}, \vec{\varphi}\rangle\rangle=\sum_{l, m=0}^{\infty} l ! m !\left\langle\Phi_{l, m}, \varphi_{l, m}\right\rangle$, where $\vec{\Phi}=\left(\Phi_{l, m}\right)_{l, m=0}^{\infty} \in G_{\theta}\left(N^{\prime} \oplus N^{\prime}\right)$ and $\vec{\varphi}=\left(\varphi_{l, m}\right)_{l, m=0}^{\infty} \in F_{\theta}(N \oplus N)$. One can easily see that $\mathscr{U}_{\theta}^{*}$ is given by

$\mathscr{U}_{\theta}^{*}=\left\{\Xi=\sum_{l, m=0}^{\infty} \Xi_{l, m}\left(\kappa_{l, m}\right),\left(\kappa_{l, m}\right)_{l, m=0}^{\infty} \in G_{\theta}\left(N^{\prime} \oplus N^{\prime}\right)\right\}$

and we have the following isomorphism

Theorem 6 The map $f_{\tau}$ defined by $f_{\tau}: \mathscr{L}\left(\mathscr{F}_{\theta}, \mathscr{F}_{\theta}^{*}\right) \longrightarrow \mathscr{U}_{\theta}^{*}, \quad \Xi \longmapsto \Xi_{\tau} \diamond \Xi$, is an isometry topological isomorphism.

Theorem 7 The Wick symbol map realizes a topological isomorphism between the space $\mathscr{U}_{\theta}^{*}$ and the space $\mathscr{G}_{\theta^{*}}(N \oplus$ $N)$. 


\section{QWN-integral kernel operator}

For $x_{1}, x_{2}, z \in N$ and $g\left(x_{1}, x_{2}\right)$ in $\mathscr{H}_{\theta}(N \oplus N)$, let

$\left(\partial_{1, z} g\right)\left(x_{1}, x_{2}\right):=\lim _{\lambda \rightarrow 0} \frac{g\left(x_{1}+\lambda z, x_{2}\right)-g\left(x_{1}, x_{2}\right)}{\lambda}$,

$\left(\partial_{2, z} g\right)\left(x_{1}, x_{2}\right):=\lim _{\lambda \rightarrow 0} \frac{g\left(x_{1}, x_{2}+\lambda z\right)-g\left(x_{1}, x_{2}\right)}{\lambda}$.

Then, in view of Theorem 2, we give in the next theorem and proposition an analytic characterization of the QWN-derivatives and their adjoints, see also [6] and [24].

Theorem 8 Let be given $z \in N$. For all $\Xi \in \mathscr{U}_{\theta}$, there exist a unique $\widetilde{\Xi}_{1, z}$ and a unique $\widetilde{\Xi}_{2, z}$ in $\mathscr{U}_{\theta}$ given by $\widetilde{\Xi}_{1, z}=\omega^{-1} \partial_{1, z} \omega(\Xi)$ and $\widetilde{\Xi}_{2, z}=\omega^{-1} \partial_{2, z} \omega(\Xi)$. Moreover, we have $D_{z}^{-} \Xi=\widetilde{\Xi}_{1, z}$ and $D_{z}^{+} \Xi=\widetilde{\Xi}_{2, z}$.

Proposition 1 For all $\Xi \in \mathscr{U}_{\theta}^{*}$ and all $z \in N$, there exist a unique $\widetilde{\Xi}_{1, z}^{*} \in \mathscr{U}_{\theta}^{*}$ and a unique $\widetilde{\Xi}_{2, z}^{*} \in \mathscr{U}_{\theta}^{*}$ given by $\widetilde{\Xi}_{1, z}^{*}=\omega^{-1} \partial_{1, z}^{*} \omega(\Xi)$ and $\widetilde{\Xi}_{2, z}^{*}=\omega^{-1} \partial_{2, z}^{*} \omega(\Xi)$ where $\partial_{1, z}^{*}$ and $\partial_{2, z}^{*}$ are given by

$\left\langle\left\langle\partial_{1, z}^{*} f, g\right\rangle\right\rangle=\left\langle\left\langle f, \partial_{1, z} g\right\rangle\right\rangle, \quad\left\langle\left\langle\partial_{2, z}^{*} f, g\right\rangle\right\rangle=\left\langle\left\langle f, \partial_{2, z} g\right\rangle\right\rangle$,

forall $g \in \mathscr{H}_{\theta}(N \oplus N)$ and $f \in \mathscr{G}_{\theta^{*}}(N \oplus N)$. Moreover, the operators $\widetilde{\Xi}_{1, z}^{*}$ and $\widetilde{\Xi}_{2, z}^{*}$ are denoted by $\left(D_{z}^{-}\right)^{*} \Xi:=\widetilde{\Xi}_{1, z}^{*}$ and $\left(D_{z}^{+}\right)^{*} \Xi:=\widetilde{\Xi}_{2, z}^{*}$

From Theorems 2, 8 and Proposition $1, D_{z}^{ \pm}$is a continuous linear operator from $\mathscr{U}_{\theta}$ into itself, $\left(D_{z}^{ \pm}\right)^{*}$ is a continuous linear operator from $\mathscr{U}_{\theta}^{*}$ into itself and in particular, the restriction of $\left(D_{z}^{ \pm}\right)^{*}$ is a continuous linear operator from $\mathscr{U}_{\theta}$ into itself.

Definition 1 Let $S=\sum_{l, m} \Xi_{l, m}\left(s_{l, m}\right)$ in $\mathscr{U}_{\theta}$ and $T=\sum_{l, m} \Xi_{l, m}\left(t_{l, m}\right)$ in $\mathscr{U}_{\theta}^{*}$, where $t_{l, m} \in\left(N^{\prime}\right)^{\widehat{\otimes} l+m}$ and $s_{l, m} \in N^{\widehat{\otimes} l+m}$. Then, the duality between the two spaces $\mathscr{U}_{\theta}$ and $\mathscr{U}_{\theta}^{*}$, denoted by $\langle\langle., .\rangle$.$\rangle , is defined as follows$

$\left\langle\langle\langle T, S\rangle\rangle:=\sum_{l, m=0}^{\infty} l ! m !\left\langle t_{l, m}, s_{l, m}\right\rangle\right.$.

Lemma 1 For $S, T \in \mathscr{U}_{\theta}$, we put

$\eta_{S, T}\left(s_{1}, \cdots, s_{j}, t_{1}, \cdots, t_{k} ; u_{1}, \cdots, u_{l}, v_{1}, \cdots, v_{m}\right)$

$:=\|\left(D_{s_{1}}^{+}\right)^{*} \cdots\left(D_{s_{j}}^{+}\right)^{*}\left(D_{t_{1}}^{-}\right)^{*} \cdots\left(D_{t_{k}}^{-}\right)^{*} D_{u_{1}}^{+} \cdots D_{u_{l}}^{+} D_{v_{1}}^{-} \cdots D_{v_{m}}^{-} S, T \rrbracket$.

Then, there exist $M(j, k, l, m)>0$ such that for any $\alpha \geq 0, \gamma_{1}, \gamma_{2}, \gamma_{3}, \gamma_{4}>0$, we have

$\left|\eta_{S, T}\right|_{\alpha} \leq M(j, k, l, m)\|S\|_{\theta, \alpha,\left(\gamma_{1}, \gamma_{2}\right)}\|T\|_{\theta, \alpha,\left(\gamma_{3}, \gamma_{4}\right)}$.

In particular, $\eta_{S, T} \in N^{\otimes(j+k+l+m)}$.

Proof Let be given $l, m \geq 0$ and $\kappa_{l, m}$ in $\left(\left(N^{\otimes l}\right) \otimes\left(N^{\otimes m}\right)\right)_{s y m(l, m)}$. For $z \in N$, by direct computation, the partial derivatives of the identity (8) in the direction $z$ are given by

$\partial_{1, z} \omega\left(\Xi_{l, m}\left(\kappa_{l, m}\right)\right)(\xi, \eta)=\omega\left(m \Xi_{l, m-1}\left(\kappa_{l, m} \otimes_{1} z\right)\right)(\xi, \eta)$

and

$\partial_{2, z} \omega\left(\Xi_{l, m}\left(\kappa_{l, m}\right)\right)(\xi, \eta)=\omega\left(l \Xi_{l-1, m}\left(z \otimes \kappa_{l, m}\right)\right)(\xi, \eta)$,

where for $z_{p} \in\left(N^{\otimes p}\right)^{\prime}$, and $\xi_{l+m-p} \in N^{\otimes(l+m-p)}, p \leq l+m$, the contractions $z_{p} \otimes_{p} \kappa_{l, m}$ and $\kappa_{l, m} \otimes^{p} z_{p}$ are given by

$\left\langle z_{p} \otimes^{p} \kappa_{l, m}, \xi_{l-p+m}\right\rangle=\left\langle\kappa_{l, m}, z_{p} \otimes \xi_{l-p+m}\right\rangle, \quad\left\langle\kappa_{l, m} \otimes_{p} z_{p}, \xi_{l+m-p}\right\rangle=\left\langle\kappa_{l, m}, \xi_{l+m-p} \otimes z_{p}\right\rangle$. 
Similarly, using Proposition 1 we get

$\partial_{1, z}^{*} \omega\left(\Xi_{l, m}\left(\kappa_{l, m}\right)\right)(\xi, \eta)=\omega\left(\Xi_{l, m+1}\left(\kappa_{l, m} \otimes z\right)\right)(\xi, \eta)$

$\partial_{2, z}^{*} \omega\left(\Xi_{l, m}\left(\kappa_{l, m}\right)\right)(\xi, \eta)=\omega\left(\Xi_{l+1, m}\left(z \otimes \kappa_{l, m}\right)\right)(\xi, \eta)$.

Now, let $S=\sum_{l, m} \Xi_{l, m}\left(s_{l, m}\right)$ and $T=\sum_{l, m} \Xi_{l, m}\left(t_{l, m}\right)$ in $\mathscr{U}_{\theta}$ where $s_{l, m}, t_{l, m} \in N^{\widehat{\otimes} l+m}$. It then follows from (16) and (17) that

$$
\begin{aligned}
& \partial_{2, \delta_{u_{1}}} \cdots \partial_{2, \delta_{u_{l}}} \partial_{1, \delta_{v_{1}}} \cdots \partial_{1, \delta_{v_{m}}} \omega(S)(\xi, \eta)= \\
& \sum_{p, q=0}^{\infty} \frac{(p+l) !}{p !} \frac{(q+m) !}{q !}\left\langle\left(\delta_{u_{1}} \widehat{\otimes} \cdots \widehat{\otimes} \delta_{u_{l}}\right) \otimes^{l} s_{p+l, q+m} \otimes_{m}\left(\delta_{v_{1}} \widehat{\otimes} \cdots \widehat{\otimes} \delta_{v_{m}}\right), \eta^{\otimes p} \otimes \xi^{\otimes q}\right\rangle
\end{aligned}
$$

and

$\partial_{2, \delta_{s_{1}}} \cdots \partial_{2, \delta_{s_{j}}} \partial_{1, \delta_{t_{1}}} \cdots \partial_{1, \delta_{t_{k}}} \omega(T)(\xi, \eta)$

$=\sum_{p, q=0}^{\infty} \frac{(p+j) !}{p !} \frac{(q+k) !}{q !}\left\langle\left(\delta_{s_{1}} \widehat{\otimes} \cdots \widehat{\otimes} \delta_{s_{j}}\right) \otimes^{j} t_{p+j, q+k} \otimes_{k}\left(\delta_{t_{1}} \widehat{\otimes} \cdots \widehat{\otimes} \delta_{t_{k}}\right), \eta^{\otimes p} \otimes \xi^{\otimes q}\right\rangle$.

Hence,

$$
\begin{aligned}
\eta_{S, T} & \left(s_{1}, \cdots, s_{j}, t_{1}, \cdots, t_{k} ; u_{1}, \cdots, u_{l}, v_{1}, \cdots, v_{m}\right) \\
= & \left\langle\partial_{2, \delta_{u_{1}}} \cdots \partial_{2, \delta_{u_{l}}} \partial_{1, \delta_{v_{1}}} \partial_{1, \delta_{v_{m}}} \omega(S), \partial_{2, \delta_{s_{1}}} \cdots \partial_{2, \delta_{s_{j}}} \partial_{\left.1, \delta_{t_{1}} \cdots \partial_{1, t_{k}} \omega(T)\right\rangle}\right. \\
= & \sum_{p, q=0}^{\infty} \frac{(p+l) !(q+m) !(p+j) !(q+k) !}{p ! q !} \\
& \left\langle\left(\delta_{u_{1}} \widehat{\otimes} \cdots \widehat{\otimes} \delta_{u_{l}}\right) \otimes s^{l} s_{p+l, q+m} \otimes_{m}\left(\delta_{v_{1}} \widehat{\otimes} \cdots \widehat{\otimes} \delta_{v_{m}}\right),\left(\delta_{s_{1}} \widehat{\otimes} \cdots \widehat{\otimes} \delta_{s_{j}}\right) \otimes^{j} t_{p+j, q+k} \otimes_{k}\left(\delta_{t_{1}} \widehat{\otimes} \cdots \widehat{\otimes} \delta_{t_{k}}\right)\right\rangle \\
= & \sum_{p, q=0}^{\infty} \frac{(p+l) !(q+m) !(p+j) !(q+k) !}{p ! q !} \\
& \left\langle\left(\delta_{s_{1}} \widehat{\otimes} \cdots \widehat{\otimes} \delta_{s_{j}}\right) \otimes\left(\delta_{t_{1}} \widehat{\otimes} \cdots \widehat{\otimes} \delta_{t_{k}}\right) \otimes\left(\delta_{u_{1}} \widehat{\otimes} \cdots \widehat{\otimes} \delta_{u_{l}}\right) \otimes\left(\delta_{v_{1}} \widehat{\otimes} \cdots \widehat{\otimes} \delta_{v_{m}}\right), t_{p+j, q+k} \otimes_{q}^{p} s_{p+l, q+m}\right\rangle .
\end{aligned}
$$

Therefore,

$\eta_{S, T}=\sum_{p, q=0}^{\infty} \frac{(p+l) !(q+m) !(p+j) !(q+k) !}{p ! q !} t_{p+j, q+k} \otimes_{q}^{p} s_{p+l, q+m}$.

Since, for $\alpha \geq 0$ and $\gamma_{1}, \gamma_{2}>0$

$\left|t_{p+j, q+k} \otimes_{q}^{p} s_{p+l, q+m}\right|_{\alpha} \leq\left|t_{p+j, q+k}\right|_{\alpha}\left|s_{p+l, q+m}\right|_{\alpha}$

we get

$$
\begin{aligned}
\left|\eta_{S, T}\right|_{\alpha} \leq & \sum_{p, q=0}^{\infty} \gamma_{1}^{\left(\frac{l}{2}\right)} \gamma_{2}^{\left(\frac{m}{2}\right)} \gamma_{4}^{\left(\frac{k}{2}\right)} \gamma_{3}^{\left(\frac{j}{2}\right)}\left(\frac{(p+l) !(p+j) !}{p !}\left(\gamma_{1} \gamma_{3}\right)^{\frac{p}{2}} \theta_{p+l} \theta_{p+j}\right) \\
& \times\left(\frac{(q+m) !(q+k) !}{q !}\left(\gamma_{2} \gamma_{4}\right)^{\frac{q}{2}} \theta_{q+m} \theta_{q+k}\right)\left(\theta_{p+l}^{-1} \theta_{q+m}^{-1} \gamma_{1}^{-\left(\frac{p+l}{2}\right)} \gamma_{2}^{-\left(\frac{q+m}{2}\right)}\left|s_{p+l, q+m}\right|_{\alpha}\right) \\
& \times\left(\theta_{p+j}^{-1} \theta_{q+k}^{-1} \gamma_{3}^{-\left(\frac{p+j}{2}\right)} \gamma_{4}^{-\left(\frac{q+k}{2}\right)}\left|t_{p+j, q+k}\right|_{\alpha}\right) \\
\leq & M(j, k, l, m)\|\vec{f}\|_{\theta, \alpha,\left(\gamma_{1}, \gamma_{2}\right)}\|\vec{g}\|_{\theta, \alpha,\left(\gamma_{3}, \gamma_{4}\right)} \\
\leq & M(j, k, l, m) K\|\omega(S)\|_{\theta, \alpha,\left(\gamma_{1}, \gamma_{2}\right)}\|\omega(T)\|_{\theta, \alpha,\left(\gamma_{3}, \gamma_{4}\right)}
\end{aligned}
$$


where $\vec{f}=\left(s_{l, m}\right)_{l, m}, \vec{g}=\left(t_{j, k}\right)_{j, k}$,

$M(j, k, l, m)=M_{1}\left(4 \sqrt{\gamma_{3}}\right)^{j}\left(4 \sqrt{\gamma_{4}}\right)^{k}\left(4 \sqrt{\gamma_{1}}\right)^{l}\left(4 \sqrt{\gamma_{2}}\right)^{m} j ! k ! l ! m ! \theta_{j} \theta_{k} \theta_{l} \theta_{m}$

and

$M_{1}=\sup _{p \geq 0}\left\{\left(16 \sqrt{\gamma_{1} \gamma_{3}}\right)^{p} p ! \theta_{p}^{2}\right\} \sup _{q \geq 0}\left\{\left(16 \sqrt{\gamma_{2} \gamma_{4}}\right)^{q} q ! \theta_{q}^{2}\right\}$,

$M_{1}<\infty$, see [23], which completes the proof.

Theorem 9 For any $\kappa \in\left(N^{\otimes(j+k+l+m)}\right)^{\prime}$ there exists a continuous linear operator $\Xi_{j, k, l, m}(\kappa) \in \mathscr{L}\left(\mathscr{U}_{\theta}, \mathscr{U}_{\theta}^{*}\right)$ such that

$\left.\left\langle\left\langle\Xi_{j, k, l, m}(\kappa) S, T\right\rangle\right\rangle\right\rangle=\left\langle\kappa, \eta_{S, T}\right\rangle, \quad S, T \in \mathscr{U}_{\theta}$,

where

$$
\begin{aligned}
& \eta_{S, T}\left(s_{1}, \cdots, s_{j}, t_{1}, \cdots, t_{k} ; u_{1}, \cdots, u_{l}, v_{1}, \cdots, v_{m}\right) \\
& =\left\|\left(D_{s_{1}}^{+}\right)^{*} \cdots\left(D_{s_{j}}^{+}\right)^{*}\left(D_{t_{1}}^{-}\right)^{*} \cdots\left(D_{t_{k}}^{-}\right)^{*} D_{u_{1}}^{+} \cdots D_{u_{l}}^{+} D_{v_{1}}^{-} \cdots D_{v_{m}}^{-} S, T\right\| .
\end{aligned}
$$

Moreover, for any $\alpha \geq 0, \gamma_{1}, \gamma_{2}>0$ with $|\kappa|_{-\alpha}<\infty$ it holds that

$\left\|\Xi_{j, k, l, m}(\kappa) S\right\|_{\theta,-\alpha,\left(\gamma_{1}, \gamma_{2}\right)} \leq M(j, k, l, m)|\kappa|_{-\alpha}\|S\|_{\theta, \alpha,\left(\gamma_{1}, \gamma_{2}\right)}$.

Proof First note that for any $\kappa \in\left(N^{\otimes(j+k+l+m)}\right)^{\prime}$,

$(S, T) \mapsto\left\langle\kappa, \eta_{S, T}\right\rangle, \quad S, T \in \mathscr{U}_{\theta}$,

is a continuous bilinear form on $\mathscr{U}_{\theta}$. In fact, by Lemma 1 we have

$$
\begin{aligned}
\left|\left\langle\kappa, \eta_{S, T}\right\rangle\right| & \leq|\kappa|_{-\alpha}\left|\eta_{S, T}\right|_{\alpha} \\
& \leq M(j, k, l, m)|\kappa|_{-\alpha}\|S\|_{\theta, \alpha,\left(\gamma_{1}, \gamma_{2}\right)}\|T\|_{\theta, \alpha,\left(\gamma_{3}, \gamma_{4}\right)},
\end{aligned}
$$

where $M(j, k, l, m)$ is given in Lemma 1 . Therefore, there is a continuous linear operator $\Xi_{j, k, l, m}(\kappa)$ in $\mathscr{L}\left(\mathscr{U}_{\theta}, \mathscr{U}_{\theta}^{*}\right)$ such that

$\left.\left\langle\left\langle\Xi_{j, k, l, m}(\kappa) S, T\right\rangle\right\rangle\right\rangle=\left\langle\kappa, \eta_{S, T}\right\rangle$.

It then follows from (21) that

$$
\begin{aligned}
\left\|\Xi_{j, k, l, m}(\kappa) S\right\|_{\theta,-\alpha,\left(\gamma_{1}, \gamma_{2}\right)} & \leq \sup \left\{\left|\left\langle\kappa, \eta_{S, T}\right\rangle\right| ;\|T\|_{\theta, \alpha,\left(\gamma_{3}, \gamma_{4}\right)}<1\right\} \\
& \leq M(j, k, l, m)|\kappa|_{-\alpha}\|S\|_{\theta, \alpha,\left(\gamma_{1}, \gamma_{2}\right)}
\end{aligned}
$$

which completes the proof.

The operator $\Xi_{j, k, l, m}(\kappa)$ is thus defined through two canonical bilinear forms:

$\left.\left\langle\left\langle\Xi_{j, k, l, m}(\kappa) S, T\right\rangle\right\rangle\right\rangle$

$$
\left.=\left\langle\kappa,\left\|\left(D_{s_{1}}^{+}\right)^{*} \cdots\left(D_{s_{j}}^{+}\right)^{*}\left(D_{t_{1}}^{-}\right)^{*} \cdots\left(D_{t_{k}}^{-}\right)^{*} D_{u_{1}}^{+} \cdots D_{u_{l}}^{+} D_{v_{1}}^{-} \cdots D_{v_{m}}^{-} S, T\right\|\right\rangle\right\rangle,
$$

where $S, T \in \mathscr{U}_{\theta}$. This suggests us to employ a formal integral expression:

$$
\begin{array}{r}
\Xi_{j, k, l, m}(\kappa)=\int_{\mathbb{R}^{j+k+l+m}} \kappa\left(s_{1}, \cdots, s_{j}, t_{1}, \cdots, t_{k} ; u_{1}, \cdots, u_{l}, v_{1}, \cdots, v_{m}\right) \\
\left(D_{s_{1}}^{+}\right)^{*} \cdots\left(D_{s_{j}}^{+}\right)^{*}\left(D_{t_{1}}^{-}\right)^{*} \cdots\left(D_{t_{k}}^{-}\right)^{*} D_{u_{1}}^{+} \cdots D_{u_{l}}^{+} D_{v_{1}}^{-} \cdots D_{v_{m}}^{-} \\
\mathrm{d} s_{1} \cdots \mathrm{d} s_{j} \mathrm{~d} t_{1} \cdots \mathrm{d} t_{k} \mathrm{~d} u_{1} \cdots \mathrm{d} u_{l} \mathrm{~d} v_{1} \cdots \mathrm{d} v_{m} .
\end{array}
$$


We call $\Xi_{j, k, l, m}$ an QWN-integral operator with kernel distribution $\kappa$. It is possible to write down the action of $\Xi_{j, k, l, m}(\kappa)$ explicitly using the contraction of tensor product.

For a later use we need to define an the operator $S^{a, b}$ as follows. For any $a, b \in N^{\prime}$

$S^{a, b} \equiv \sum_{l, m=0}^{\infty} \Xi_{l, m}\left(\kappa_{l, m}(a, b)\right) \in \mathscr{U}_{\theta}^{*}$,

where $\kappa_{l, m}(a, b)=\frac{1}{l ! m !} a^{\otimes l} \otimes b^{\otimes m}$. It is noteworthy that $\left\{S^{a, b} ; a, b \in N^{\prime}\right\}$ spans a dense subspace of $\mathscr{U}_{\theta}^{*}$ and $\left\{S^{a, b} ; a, b \in N\right\}$ spans a dense subspace of $\mathscr{U}_{\theta}$.

Proposition 2 Let $S \in \mathscr{U}_{\theta}$ given by $S=\sum_{p, q=0}^{\infty} \Xi_{p, q}\left(s_{p, q}\right)$. Then, for $\kappa \in\left(N^{\otimes(j+k+l+m)}\right)^{\prime}$ we have

$\Xi_{j, k, l, m}(\kappa) S=\sum_{p, q=0}^{\infty} \frac{(p+l) !(q+m) !}{p ! q !} S_{p+j, q+k}\left(\kappa \otimes_{m}^{l} s_{p+l, q+m}\right)$.

In particular, for $a, b \in N$ we have

$\Xi_{j, k, l, m}(\kappa) S^{a, b}=\sum_{p, q=0}^{\infty} \frac{1}{p ! q !} S_{p+k, q+j}\left(\left(\kappa \otimes_{m}^{l}\left(a^{\otimes l} \otimes b^{\otimes m}\right)\right) \otimes\left(a^{\otimes p} \otimes b^{\otimes q}\right)\right)$.

Proof Let $T \in \mathscr{U}_{\theta}$ be given as $T=\sum_{p, q=0}^{\infty} \Xi_{p, q}\left(t_{p, q}\right)$. Then, by definition,

$$
\begin{aligned}
\left\langle\left\langle\Xi_{j, k, l, m}(\kappa) S, T\right\rangle\right\rangle & =\sum_{p, q=0}^{\infty} \frac{(p+l) !(q+m) !(p+j) !(q+k) !}{p ! q !}\left\langle\kappa, t_{p+j, q+k} \otimes_{q}^{p} s_{p+l, q+m}\right\rangle \\
& =\sum_{p, q=0}^{\infty} \frac{(p+l) !(q+m) !(p+j) !(q+k) !}{p ! q !}\left\langle\kappa \otimes_{m}^{l} s_{p+l, q+m}, t_{p+j, q+k}\right\rangle,
\end{aligned}
$$

from which (23) follows. The proof of (24) is then immediate.

During the above discussion we have obtained a linear map

$\left(N^{\otimes(j+k+l+m)}\right)^{\prime} \ni \kappa \longmapsto \Xi_{j, k, l, m}(\kappa) \in \mathscr{L}\left(\mathscr{U}_{\theta}, \mathscr{U}_{\theta}^{*}\right)$.

But this not injective, namely, the kernel distribution is not uniquely determined.

For the uniqueness, we need a "partially symmetrized" kernel. We put

$\widehat{\kappa}=\frac{1}{j ! k ! l ! m !} \sum_{\pi \in G_{j} \times G_{k} \times G_{l} \times G_{m}} \kappa^{\pi}, \quad \kappa \in\left(N^{\otimes(j+k+l+m)}\right)^{\prime}$,

where.$^{\pi}$ is defined by (see[21]), for $F \in\left(N^{\otimes n}\right)^{\prime}$ and $\pi \in G_{n}$

$\left\langle F^{\pi}, \xi_{1} \otimes \cdots \otimes \xi_{n}\right\rangle=\left\langle F, \xi_{\pi^{-1}(1)} \otimes \cdots \otimes \xi_{\pi^{-1}(n)}\right\rangle, \quad \xi_{1}, \cdots, \xi_{n} \in N$.

We first note the following

Proposition 3 For all $\kappa \in\left(N^{\otimes(j+k+l+m)}\right)^{\prime}$ we have

$\Xi_{j, k, l, m}(\kappa)=\Xi_{j, k, l, m}(\widehat{\kappa})$.

Proof Since $\boldsymbol{\Xi}_{j, k, l, m}(\kappa)$ is defined uniquely by (22), the assertion follows immediately from the fact that $\left[D_{s}^{ \pm}, D_{t}^{ \pm}\right]=0$ and $\left[\left(D_{s}^{ \pm}\right)^{*},\left(D_{t}^{ \pm}\right)^{*}\right]=0, s, t \in \mathbb{R}$,

which can be shown by a straight forward calculus. 
Proposition 4 Let $\kappa \in\left(N^{\otimes(j+k+l+m)}\right)^{\prime}$. Then $\Xi_{j, k, l, m}(\kappa)=0$ if and only if $\widehat{\kappa}=0$. In other words, for $\kappa \in$ $\left(N^{\otimes(j+k+l+m)}\right)_{s y m(j, k, l, m)}^{\prime}$, the map

$\kappa \longmapsto \Xi_{j, k, l, m}(\kappa) \in \mathscr{L}\left(\mathscr{U}_{\theta}, \mathscr{U}_{\theta}^{*}\right)$

is injective.

Proof Suppose that $\widehat{\kappa}=0$. Then, we see from Proposition 3 that $\Xi_{j, k, l, m}(\kappa)=\Xi_{j, k, l, m}(\widehat{\kappa})=0$. Conversely, we suppose that $\Xi_{j, k, l, m}(\kappa)=0$. Consider a particular operator:

$S_{l, m}^{a, b}=\Xi_{l, m}\left(a^{\otimes l} \otimes b^{\otimes m}\right), \quad T_{j, k}^{c, d}=\Xi_{j, k}\left(c^{\otimes j} \otimes d^{\otimes k}\right), \quad a, b, c, d \in N$.

Then, it follows from the definition that

$\left\|\Xi_{j, k, l, m}(\kappa) S_{l, m}^{a, b}, T_{j, k}^{c, d}\right\|=j ! k ! l ! m !\left|\kappa, c^{\otimes j} \otimes d^{\otimes k} \otimes a^{\otimes l} \otimes b^{\otimes m}\right\rangle$.

Since $\Xi_{j, k, l, m}(\kappa)=0$ by assumption,

$0=\left\langle\kappa, c^{\otimes j} \otimes d^{\otimes k} \otimes a^{\otimes l} \otimes b^{\otimes m}\right\rangle=\left\langle\widehat{\kappa}, c^{\otimes j} \otimes d^{\otimes k} \otimes a^{\otimes l} \otimes b^{\otimes m}\right\rangle$

This being true for all, $a, b, c, d \in N$, we conclude that $\widehat{\kappa}=0$.

Proposition 5 Let $\kappa \in\left(N^{\otimes(j+k+l+m)}\right)^{\prime}$ and $\kappa^{\prime} \in\left(N^{\otimes\left(j^{\prime}+k^{\prime}+l^{\prime}+m^{\prime}\right)}\right)^{\prime}$. If $\Xi_{j, k, l, m}(\kappa)=\Xi_{j^{\prime}, k^{\prime}, l^{\prime}, m^{\prime}}\left(\kappa^{\prime}\right) \neq 0$, then $j=j^{\prime}, k=k^{\prime}, l=l^{\prime}, m=m^{\prime}$ and $\widehat{\kappa}=\widehat{\kappa}^{\prime}$.

Proof Suppose that $\Xi_{j, k, l, m}(\kappa)=\Xi_{j^{\prime}, k^{\prime}, l^{\prime}, m^{\prime}}\left(\kappa^{\prime}\right) \neq 0$. In particular, since $\Xi_{j, k, l, m}(\kappa) \neq 0$, it follows from Proposition 4 that $\widehat{\kappa} \neq 0$ and therefore, there exist $a, b, c, d \in N$ such that

$\left\langle\kappa, c^{\otimes j} \otimes d^{\otimes k} \otimes a^{\otimes l} \otimes b^{\otimes m}\right\rangle \neq 0$.

Then, we have

$\left\|\Xi_{j, k, l, m}(\kappa) S_{l, m}^{a, b}, T_{j, k}^{c, d}\right\| \neq 0$.

On the other hand, unless $j^{\prime} \leq j, k^{\prime} \leq k, l^{\prime} \leq l$ and $m^{\prime} \leq m$,

$$
\left\|\Xi_{j^{\prime}, k^{\prime}, l^{\prime}, m^{\prime}}\left(\kappa^{\prime}\right) S_{l, m}^{a, b}, T_{j, k}^{c, d}\right\|=\left\langle\kappa^{\prime},\left\|D_{u_{1}}^{+} \cdots D_{u_{l^{\prime}}}^{+} D_{v_{1}}^{-} \cdots D_{v_{m^{\prime}}}^{-} S_{l, m}^{a, b}, D_{s_{1}}^{+} \cdots D_{s_{j^{\prime}}}^{+} D_{t_{1}}^{-} \cdots D_{t_{k^{\prime}}}^{-} T_{j, k}^{c, d}\right\|\right\rangle=0 .
$$

Therefore, to have (25) it is necessary that $j^{\prime} \leq j, k^{\prime} \leq k, l^{\prime} \leq l$ and $m \leq m^{\prime}$. A similar argument with $\Xi_{j^{\prime}, k^{\prime}, l^{\prime}, m^{\prime}}\left(\kappa^{\prime}\right) \neq 0$ implies that $j \leq j^{\prime}, k \leq k^{\prime}, l \leq l^{\prime}$ and $m \leq m^{\prime}$. Hence $j^{\prime}=j, k^{\prime}=k, l^{\prime}=l$ and $m=m^{\prime}$. We then see from Proposition 4 that $\widehat{\kappa}=\widehat{\kappa^{\prime}}$.

With each $\left(\kappa_{j, k, l, m}\right)_{j, k, l, m=0}^{\infty} \in \bigoplus_{j, k, l, m=0}^{\infty}\left(N^{\otimes(j+k+l+m)}\right)_{s y m(j, k, l, m)}^{\prime}$ (algebraic direct sum) we may associated an operator

$$
\sum_{j, k, l, m=0}^{\infty} \Xi_{j, k, l, m}\left(\kappa_{j, k, l, m}\right) \in \mathscr{L}\left(\mathscr{U}_{\theta}, \mathscr{U}_{\theta}^{*}\right) .
$$

It then follows from Propositions 4 and 5 that we have a linear injection:

$\bigoplus_{j, k, l, m=0}^{\infty}\left(N^{\otimes(j+k+l+m)}\right)_{\operatorname{sym}(j, k, l, m)}^{\prime} \longrightarrow \mathscr{L}\left(\mathscr{U}_{\theta}, \mathscr{U}_{\theta}^{*}\right)$. 


\section{QWN-symbol map}

Definition 2 For $P \in \mathscr{L}\left(\mathscr{U}_{\theta}, \mathscr{U}_{\theta}^{*}\right)$, we define $\sigma^{Q}(P)$ to be the four-variable function given by $\sigma^{Q}(P)(a, b, c, d):=\left\langle\left\langle\left\langle P S^{a, b}, S^{c, d}\right\rangle\right\rangle ; \quad a, b, c, d \in N\right.$.

$\sigma^{Q}$ is referred to us a QWN-version of the usual symbol map $\sigma$.

Note that $\sigma^{Q}(I)$ is given by

$$
\begin{aligned}
\sigma^{Q}(I)(a, b, c, d) & =\left\langle S^{a, b}, S^{c, d}\right\rangle \\
& =\sum_{l, m=0}^{\infty} l ! m !\left|\frac{a^{\otimes l}}{l !} \otimes \frac{b^{\otimes m}}{m !}, \frac{c^{\otimes l}}{l !} \otimes \frac{d^{\otimes m}}{m !}\right\rangle \\
& =\exp \{\langle a, c\rangle+\langle b, d\rangle\} .
\end{aligned}
$$

As in [10] and [17], we can define

$$
\mathscr{G}_{\theta}\left(N^{4}\right)=\bigcup_{p_{1}, p_{2}, p_{3}, p_{4} \geq 0, m_{1}, m_{2}, m_{3}, m_{4}>0} \operatorname{Exp}\left(N_{p_{1}} \oplus N_{p_{2}} \oplus N_{p_{3}} \oplus N_{p_{4}}, \theta, m_{1}, m_{2}, m_{3}, m_{4}\right) .
$$

Theorem 10 The map $\sigma^{Q}$ is a topological isomorphism from $\mathscr{L}\left(\mathscr{U}_{\theta}, \mathscr{U}_{\theta}^{*}\right)$ into $\mathscr{G}_{\theta^{*}}\left(N^{4}\right)$.

Proof By the kernel theorem, we have

$$
\begin{aligned}
\mathscr{L}\left(\mathscr{U}_{\theta}, \mathscr{U}_{\theta}^{*}\right) & \cong \mathscr{L}\left(\mathscr{L}\left(\mathscr{F}_{\theta}^{*}, \mathscr{F}_{\theta}\right), \mathscr{L}\left(\mathscr{F}_{\theta}, \mathscr{F}_{\theta}^{*}\right)\right) \\
& \cong \mathscr{L}\left(\mathscr{F}_{\theta} \otimes \mathscr{F}_{\theta}, \mathscr{F}_{\theta}^{*} \otimes \mathscr{F}_{\theta}^{*}\right) \\
& \cong \mathscr{F}_{\theta}^{*}\left(N^{\prime} \oplus N^{\prime}\right) \otimes \mathscr{F}_{\theta}^{*}\left(N^{\prime} \oplus N^{\prime}\right) \\
& \cong \mathscr{G}_{\theta^{*}}(N \oplus N) \otimes \mathscr{G}_{\theta^{*}}(N \oplus N)
\end{aligned}
$$

so that the QWN-symbol map can be seen as a composition of topological isomorphisms.

The QWN-symbol of a QWN-integral kernel QWN-operator is given in the following.

Proposition 6 Let $\kappa \in\left(N^{\otimes(j+k+l+m)}\right)^{\prime}$. Then, for $a, b, c, d \in N$

$$
\begin{aligned}
\sigma^{Q}\left(\Xi_{j, k, l, m}(\kappa)\right)(a, b, c, d) & =\| \Xi_{j, k, l, m}(\kappa) S^{a, b}, S^{c, d} \rrbracket \\
& =\left\langle\kappa, c^{\otimes j} \otimes d^{\otimes k} \otimes a^{\otimes l} \otimes b^{\otimes m}\right\rangle e^{\langle a, c\rangle} e^{\langle b, d\rangle} .
\end{aligned}
$$

Proof By Proposition 2 we have

$$
\begin{aligned}
\Xi_{j, k, l, m}(\kappa) S^{a, b} & =\sum_{p, q=0}^{\infty} \frac{1}{p ! q !} S_{p+j, q+k}\left(\left(\kappa \otimes_{m}^{l}\left(a^{\otimes l} \otimes b^{\otimes m}\right)\right) \otimes\left(a^{\otimes p} \otimes b^{\otimes q}\right)\right) \\
& =\sum_{p=j, q=k}^{\infty} \frac{1}{(p-j) !(q-k) !} \Xi_{p, q}\left(\left(\kappa \otimes_{m}^{l}\left(a^{\otimes l} \otimes b^{\otimes m}\right)\right) \otimes\left(a^{\otimes(p-j)} \otimes b^{\otimes(q-k)}\right)\right) .
\end{aligned}
$$

On the other hand,

$S^{c, d}=\sum_{p, q=0}^{\infty} S_{p, q}\left(\frac{c^{\otimes^{p}}}{p !} \otimes \frac{d^{\otimes^{q}}}{q !}\right)$. 
Hence

$$
\begin{aligned}
\left.\left\langle\left\langle\Xi_{j, k, l, m}(\kappa) S^{a, b}, S^{c, d}\right\rangle\right\rangle\right\rangle= & \sum_{p=j, q=k}^{\infty} p ! q ! \frac{1}{(p-j) !} \frac{1}{(q-k) !} \\
& \left\langle\left(\left(\kappa \otimes_{m}^{l}\left(a^{\otimes l} \otimes b^{\otimes m}\right)\right) \otimes\left(a^{\otimes(p-j)} \otimes b^{\otimes(q-k)}\right)\right), \frac{c^{\otimes p}}{p !} \otimes \frac{d^{\otimes q}}{q !}\right\rangle \\
= & \sum_{p=j, q=k}^{\infty} \frac{1}{(p-j) !} \frac{1}{(q-k) !} \\
& \left\langle\left(\kappa \otimes_{m}^{l}\left(a^{\otimes l} \otimes b^{\otimes m}\right)\right), c^{\otimes j} \otimes d^{\otimes k}\right\rangle\langle a, c\rangle^{p-j}\langle b, d\rangle^{q-k} \\
= & \left\langle\kappa, c^{\otimes j} \otimes d^{\otimes k} \otimes a^{\otimes l} \otimes b^{\otimes m}\right\rangle e^{\langle a, c\rangle} e^{\langle b, d\rangle .}
\end{aligned}
$$

This completes the proof.

\section{Chaotic expansion of QWN-operators:}

Given $\Xi^{Q} \in \mathscr{L}\left(\mathscr{U}_{\theta}, \mathscr{U}_{\theta}^{*}\right)$, we consider the Taylor expansion of $\sigma^{Q}\left(\Xi^{Q}\right)$ in $\mathscr{G}_{\theta^{*}}\left(N^{4}\right)$ :

$\sigma^{Q}\left(\Xi^{Q}\right)(a, b, c, d)=\sum_{j, k, l, m}\left\langle\lambda_{j, k, l, m}, c^{\otimes j} \otimes d^{\otimes k} \otimes a^{\otimes l} \otimes b^{\otimes m}\right\rangle, \quad \lambda_{j, k, l, m} \in\left(N^{\otimes(j+k+l+m)}\right)^{\prime}$.

It is obvious by Theorem 10 that there exists $\Xi_{j, k, l, m} \in \mathscr{L}\left(\mathscr{U}_{\theta}, \mathscr{U}_{\theta}^{*}\right)$ such that

$\sigma^{Q}\left(\Xi_{j, k, l, m}\left(\lambda_{j, k, l, m}\right)\right)(a, b, c, d)=\left\langle\lambda_{j, k, l, m}, c^{\otimes j} \otimes d^{\otimes k} \otimes a^{\otimes l} \otimes b^{\otimes m}\right\rangle$.

Thus, we come to

$\Xi^{Q}=\sum_{j, k, l, m} \Xi_{j, k, l, m}\left(\lambda_{j, k, l, m}\right)$

which is called the chaotic expansion of $\Xi^{Q} \in \mathscr{L}\left(\mathscr{U}_{\theta}, \mathscr{U}_{\theta}^{*}\right)$.

Lemma 2 For each $\kappa \in\left(N^{\otimes(j+k+l+m)}\right)^{\prime}$ there exists a QWN-operator $\Xi_{j, k, l, m}(\kappa)$ in $\mathscr{L}\left(\mathscr{U}_{\theta}, \mathscr{U}_{\theta}^{*}\right)$ whose symbol is given by

$$
\begin{aligned}
\sigma^{Q}\left(\Xi_{j, k, l, m}(\kappa)\right)(a, b, c, d) & =\| \Xi_{j, k, l, m}(\kappa) S^{a, b}, S^{c, d} \rrbracket \\
& =\left\langle\kappa, c^{\otimes j} \otimes d^{\otimes k} \otimes a^{\otimes l} \otimes b^{\otimes m}\right\rangle e^{\langle a, c\rangle} e^{\langle b, d\rangle}, \quad a, b, c, d \in N .
\end{aligned}
$$

Proof We write $\Theta(a, b, c, d)$ for the righthand side of (26). It is sufficient to show that $\Theta \in \mathscr{G}_{\theta^{*}}\left(N^{4}\right)$ by Theorem 10. Since $\left\langle\kappa, c^{\otimes j} \otimes d^{\otimes k} \otimes a^{\otimes l} \otimes b^{\otimes m}\right\rangle$ is of polynomial growth, it belongs to $\mathscr{G}_{\theta^{*}}\left(N^{4}\right)$. From the fact $\mathscr{U}_{\theta} \subset \mathscr{U}_{\theta}^{*}$ we see that the identity operator I on $\mathscr{U}_{\theta}$ is a member of $\mathscr{L}\left(\mathscr{U}_{\theta}, \mathscr{U}_{\theta}^{*}\right)$, and hence $\sigma^{Q}(I) \in \mathscr{G}_{\theta^{*}}\left(N^{4}\right)$. Note that $\sigma^{Q}(I)(a, b, c, d)=\left\langle\left\langle\left\langle S^{a, b}, S^{c, d}\right\rangle\right\rangle\right\rangle=e^{\langle a, c\rangle} e^{\langle b, d\rangle}$.

Since $\mathscr{G}_{\theta^{*}}\left(N^{4}\right)$ is closed under pointwise multiplication, we conclude that $\Theta \in \mathscr{G}_{\theta^{*}}\left(N^{4}\right)$.

Theorem 11 Let $\Theta: N^{4} \rightarrow \mathbb{C}$ belongs to $\mathscr{G}_{\theta^{*}}\left(N^{4}\right)$. Then, there exists a unique family of kernel distributions $\left\{\kappa_{j, k, l, m}\right\}_{j, k, l, m=0}^{\infty}$, where $\kappa_{j, k, l, m} \in\left(N^{\otimes(j+k+l+m)}\right)_{s y m(j, k, l, m)}^{\prime}$, such that

$\left|\kappa_{j, k, l, m}\right|_{-(\alpha+1)} \leq K(2 e \delta)^{j+k+l+m}\left(\gamma_{1}+1\right)^{j}\left(\gamma_{2}+1\right)^{k}\left(\gamma_{3}+1\right)^{l}\left(\gamma_{4}+1\right)^{m} \theta_{j}^{*} \theta_{k}^{*} \theta_{l}^{*} \theta_{m}^{*}$

for some $K, \gamma_{1}, \gamma_{2}, \gamma_{3}, \gamma_{4}>0$ and

$\Theta(a, b, c, d)=\sum_{j, k, l, m=0}^{\infty}\left\|\Xi_{j, k, l, m}\left(\kappa_{j, k, l, m}\right) S^{a, b}, S^{c, d}\right\|, \quad a, b, c, d \in N$. 
Moreover, the series

$\Xi^{Q} S=\sum_{j, k, l, m=0}^{\infty} \Xi_{j, k, l, m}\left(\kappa_{j, k, l, m}\right) S, \quad S \in \mathscr{U}_{\theta}$,

converges in $\mathscr{U}_{\theta}, \Xi^{Q} \in \mathscr{L}\left(\mathscr{U}_{\theta}, \mathscr{U}_{\theta}^{*}\right)$ and $\sigma^{Q}\left(\Xi^{Q}\right)=\Theta$.

For the proof we need some technical results.

Lemma 3 Let $f$ be an entire holomorphic function on $\mathbb{C}^{4}$ with the Taylor expansion $f(z, y, x, w)=$ $\sum_{j, k, l, m=0}^{\infty} a_{j k l m} z^{j} y^{k} x^{l} w^{m}$. Assume that

$|f(z, y, x, w)| \leq K \exp \left\{\theta^{*}\left(K_{1}|z|\right)+\theta^{*}\left(K_{2}|y|\right)+\theta^{*}\left(K_{3}|x|\right)+\theta^{*}\left(K_{4}|w|\right)\right\}, \quad z, y, x, w \in \mathbb{C}$,

for some $K \geq 0, K_{1} \geq 0, K_{2} \geq 0, K_{3} \geq 0$ and $K_{4} \geq 0$. Then

$\left|a_{j k l m}\right| \leq K K_{1}^{j} \theta_{j}^{*} K_{2}^{k} \theta_{k}^{*} K_{3}^{l} \theta_{l}^{*} K_{4}^{m} \theta_{m}^{*}$.

Proof Similar to the classical case (see[10,21]). Since for $R_{1}>0, R_{2}>0, R_{3}>0$ and $R_{4}>0$

$a_{j k l m}=\left(\frac{1}{2 \Pi i}\right)^{4} \int_{|z|=R_{1}} \int_{|y|=R_{2}} \int_{|x|=R_{3}} \int_{|w|=R_{4}} \frac{f(z, y, x, w)}{z^{j+1} y^{k+1} x^{l+1} w^{m+1}} \mathrm{~d} z \mathrm{~d} y \mathrm{~d} x \mathrm{~d} w$

we have

$$
\begin{aligned}
\left|a_{j k l m}\right| & \leq \sup \left\{|f(z, y, s, w)| R_{1}^{-j} R_{2}^{-k} R_{3}^{-l} R_{4}^{-m} ;|z|=R_{1}, \quad|y|=R_{2}, \quad|x|=R_{3}, \quad|w|=R_{4}\right\} \\
& \leq K R_{1}^{-j} e^{\theta^{*}\left(K_{1} R_{1}\right)} R_{2}^{-k} e^{\theta^{*}\left(K_{2} R_{2}\right)} R_{3}^{-l} e^{\theta^{*}\left(K_{3} R_{3}\right)} R_{4}^{-m} e^{\theta^{*}\left(K_{4} R_{4}\right)} \\
& =K K_{1}^{j} \theta_{j}^{*} K_{2}^{k} \theta_{k}^{*} K_{3}^{l} \theta_{l}^{*} K_{4}^{m} \theta_{m}^{*} .
\end{aligned}
$$

Hence, we obtain the desired estimate.

Lemma 4 Let $\Theta$ be a $\mathbb{C}$-valued function on $N^{4}$ and assume that $\Theta \in \mathscr{G}_{\theta^{*}}\left(N^{4}\right)$. Put

$\psi(a, b, c, d)=e^{-\langle a, c\rangle} e^{-\langle b, d\rangle} \Theta(a, b, c, d), \quad a, b, c, d \in N$,

and

$$
\begin{aligned}
& \kappa_{j, k, l, m}\left(a_{1}, \cdots, a_{j}, b_{1}, \cdots, b_{k}, c_{1}, \cdots, c_{l}, d_{1}, \cdots, d_{m}\right)= \\
& \frac{1}{j ! k ! l ! m !} D_{a_{1}}^{(1)} \cdots D_{a_{j}}^{(1)} D_{b_{1}}^{(2)} \cdots D_{b_{k}}^{(2)} D_{c_{1}}^{(3)} \cdots D_{c_{l}}^{(3)} D_{d_{1}}^{(4)} \cdots D_{d_{m}}^{(4)} \psi(0,0,0,0),
\end{aligned}
$$

where

$D_{a_{1}}^{(1)} \psi(a, b, c, d)=\left.\frac{\mathrm{d}}{\mathrm{d} z}\right|_{z=0} \psi\left(z a_{1}+a, b, c, d\right), \quad D_{b_{1}}^{(2)} \psi(a, b, c, d)=\left.\frac{\mathrm{d}}{\mathrm{d} y}\right|_{y=0} \psi\left(a, y b_{1}+b, c, d\right)$,

$D_{c_{1}}^{(3)} \psi(a, b, c, d)=\left.\frac{\mathrm{d}}{\mathrm{d} x}\right|_{x=0} \psi\left(a, b, x c_{1}+c, d\right), \quad D_{d_{1}}^{(4)} \psi(a, b, c, d)=\left.\frac{\mathrm{d}}{\mathrm{d} w}\right|_{w=0} \psi\left(a, b, c, w d_{1}+d\right)$.

Then, $\kappa_{j, k, l, m}$ is a continuous $(j+k+l+m)$-linear form on $N$ with $\widehat{\kappa}_{j, k, l, m}=\kappa_{j, k, l, m}$. Moreover, there exist $K, \gamma_{1}, \gamma_{2}, \gamma_{3}, \gamma_{4}>0$ such that

$\left|\kappa_{j, k, l, m}\right|_{-(\alpha+1)} \leq K(2 e \delta)^{j+k+l+m}\left(\gamma_{1}+1\right)^{j}\left(\gamma_{2}+1\right)^{k}\left(\gamma_{3}+1\right)^{l}\left(\gamma_{4}+1\right)^{m} \theta_{j}^{*} \theta_{k}^{*} \theta_{l}^{*} \theta_{m}^{*}$.

Proof It is obvious that $\kappa_{j, k, l, m}$ is a $\mathbb{C}$-valued $(j+k+l+m)$-form on $\mathrm{N}$. We now put

$A_{j, k, l, m}(a, b, c, d)=\kappa_{j, k, l, m}(\underbrace{a, \cdots, a}_{j \text { times }}, \underbrace{b, \cdots, b}_{k \text { times }}, \underbrace{c, \cdots, c}_{l \text { times }}, \underbrace{d, \cdots, d}_{m \text { times }})$. 
Then, we have the Taylor expansion:

$$
\begin{aligned}
\psi(z a, y b, x c, w d) & =\left.\sum_{j, k, l, m=0}^{\infty} \frac{\partial^{j+k+l+m}}{\partial z^{j} \partial y^{k} \partial x^{l} \partial w^{m}} \psi(z a, y b, x c, w d)\right|_{z=y=x=w=0} \frac{z^{j} y^{k} x^{l} w^{m}}{j ! k ! l ! m !} \\
& =\sum_{j, k, l, m=0}^{\infty} A_{j, k, l, m}(a, b, c, d) z^{j} y^{k} x^{l} w^{m} .
\end{aligned}
$$

For $\rho_{1}, \rho_{2}>0$ we have

$|\langle z a, x c\rangle| \leq \frac{\rho_{1}^{2}}{2}|z|^{2}|a|_{\alpha}^{2}+\frac{1}{2 \rho_{1}^{2}}|x|^{2}|c|_{\alpha}^{2}$,

$|\langle y b, w d\rangle| \leq \frac{\rho_{2}^{2}}{2}|y|^{2}|b|_{\alpha}^{2}+\frac{1}{2 \rho_{2}^{2}}|w|^{2}|d|_{\alpha}^{2}$.

Then from the facts that $\theta \in \mathscr{G}_{\theta^{*}}\left(N^{4}\right)$ and $\theta^{*}(s)+\theta^{*}(t) \leq \theta^{*}(2 s+2 t)$, there exist $K \geq 0, \gamma_{1}, \gamma_{2}, \gamma_{3}, \gamma_{4}>0$ such that

$$
\begin{aligned}
|\psi(z a, y b, x c, w d)|= & \left|e^{-\langle z a, x c\rangle} e^{-\langle y b, w d\rangle}\right||\Theta(z a, y b, x c, w d)| \\
\leq & K \exp \left\{\frac{\rho_{1}^{2}}{2}|z|^{2}|a|_{\alpha}^{2}+\frac{1}{2 \rho_{1}^{2}}|x|^{2}|c|_{\alpha}^{2}+\frac{\rho_{2}^{2}}{2}|y|^{2}|b|_{\alpha}^{2}+\frac{1}{2 \rho_{2}^{2}}|w|^{2}|d|_{\alpha}^{2}\right\} \\
& \times \exp \left\{\theta^{*}\left(\gamma_{1}|z||a|_{\alpha}\right)+\theta^{*}\left(\gamma_{2}|y||b|_{\alpha}\right)+\theta^{*}\left(\gamma_{3}|x||c|_{\alpha}\right)+\theta^{*}\left(\gamma_{4}|w||d|_{\alpha}\right)\right\} \\
\leq & K \exp \left\{\theta^{*}\left(2\left(\gamma_{1}+1\right)|z||a|_{\alpha}\right)+\theta^{*}\left(2\left(\gamma_{2}+1\right)|y||b|_{\alpha}\right)\right. \\
& \left.+\theta^{*}\left(2\left(\gamma_{3}+1\right)|x||c|_{\alpha}\right)+\theta^{*}\left(2\left(\gamma_{4}+1\right)|w||d|_{\alpha}\right)\right\} .
\end{aligned}
$$

It then follows from Lemma 3 that

$$
\left|A_{j, k, l, m}\right| \leq K\left\{\left(2 \gamma_{1}+2\right)^{j}\left(2 \gamma_{2}+2\right)^{k}\left(2 \gamma_{3}+2\right)^{l}\left(2 \gamma_{4}+2\right)^{m} \theta_{j}^{*} \theta_{k}^{*} \theta_{l}^{*} \theta_{m}^{*}\right\}|a|_{\alpha}^{j}|b|_{\alpha}^{k}|c|_{\alpha}^{l}|d|_{\alpha}^{m} \text {. }
$$

By virtue of the polarization formula (see[21], [19]) we obtain

$$
\begin{aligned}
& \sup \left\{\left|\kappa_{j, k, l, m}\left(a_{1}, \cdots, a_{j}, b_{1}, \cdots, b_{k}, c_{1}, \cdots, c_{l}, d_{1}, \cdots, d_{m}\right)\right| ;\left|a_{1}\right|_{\alpha} \leq 1, \cdots,\left|a_{j}\right|_{\alpha} \leq 1,\right. \\
& \left.\left|b_{1}\right| \leq 1, \cdots,\left|b_{k}\right| \leq 1,\left|c_{1}\right|_{\alpha} \leq 1, \cdots,\left|c_{l}\right|_{\alpha} \leq 1,\left|d_{1}\right|_{\alpha} \leq 1, \cdots,\left|d_{m}\right|_{\alpha} \leq 1\right\} \\
\leq & K\left(2 e \gamma_{1}+2 e\right)^{j}\left(2 e \gamma_{2}+2 e\right)^{k}\left(2 e \gamma_{3}+2 e\right)^{l}\left(2 e \gamma_{4}+2 e\right)^{m} \theta_{j}^{*} \theta_{k}^{*} \theta_{l}^{*} \theta_{m}^{*} .
\end{aligned}
$$

Then, we have

$$
\begin{aligned}
& \left|\left\langle\kappa_{j, k, l, m}, e\left(i^{(1)}\right) \otimes e\left(i^{(2)}\right) \otimes e\left(i^{(3)}\right) \otimes e\left(i^{(4)}\right)\right\rangle\right|^{2} \\
& \quad \leq K^{2}\left\{\left(2 e \gamma_{1}+2 e\right)^{j}\left(2 e \gamma_{2}+2 e\right)^{k}\left(2 e \gamma_{3}+2 e\right)^{l}\left(2 e \gamma_{4}+2 e\right)^{m} \theta_{j}^{*} \theta_{k}^{*} \theta_{l}^{*} \theta_{m}^{*}\right\}^{2} \\
& \quad \times\left|e\left(i^{(1)}\right)\right|_{\alpha}\left|e\left(i^{(2)}\right)\right|_{\alpha}\left|e\left(i^{(3)}\right)\right|_{\alpha}\left|e\left(i^{(4)}\right)\right|_{\alpha}
\end{aligned}
$$

where $e\left(i^{(1)}\right)=e_{i_{1}^{(1)}} \otimes \cdots \otimes e_{i_{j}^{(1)}}, e\left(i^{(2)}\right)=e_{i_{1}^{(2)}} \otimes \cdots \otimes e_{i_{k}^{(2)}}, e\left(i^{(3)}\right)=e_{i_{1}^{(3)}} \otimes \cdots \otimes e_{i_{l}^{(3)}}$ and $e\left(i^{(4)}\right)=e_{i_{1}^{(4)}} \otimes \cdots \otimes e_{i_{m}^{(4)}}$.

Then we get

$$
\begin{aligned}
\left|\kappa_{j, k, l, m}\right|_{-(\alpha+1)}^{2}= & \sum_{i^{(1)}, i^{(2)}, i^{(3)}, i^{(4)}}\left|\left\langle\kappa_{j, k, l, m}, e\left(i^{(1)}\right) \otimes e\left(i^{(2)}\right) \otimes e\left(i^{(3)}\right) \otimes e\left(i^{(4)}\right)\right\rangle\right|^{2} \\
& \left|e\left(i^{(1)}\right)\right|_{-(\alpha+1)}^{2}\left|e\left(i^{(2)}\right)\right|_{-(\alpha+1)}^{2}\left|e\left(i^{(3)}\right)\right|_{-(\alpha+1)}^{2}\left|e\left(i^{(4)}\right)\right|_{-(\alpha+1)}^{2} \\
\leq & K^{2}\left\{\left(2 e \gamma_{1}+2 e\right)^{j}\left(2 e \gamma_{2}+2 e\right)^{k}\left(2 e \gamma_{3}+2 e\right)^{l}\left(2 e \gamma_{4}+2 e\right)^{m} \theta_{j}^{*} \theta_{k}^{*} \theta_{l}^{*} \theta_{m}^{*}\right\}^{2} \\
& \sum_{i^{(1)}, i^{(2)}, i^{(3)}, i^{(4)}}\left|e\left(i^{(1)}\right)\right|_{-1}^{2}\left|e\left(i^{(2)}\right)\right|_{-1}^{2}\left|e\left(i^{(3)}\right)\right|_{-1}^{2}\left|e\left(i^{(4)}\right)\right|_{-1}^{2} \\
\leq & \left\{K\left(2 e \delta \gamma_{1}+2 e \delta\right)^{j}\left(2 e \delta \gamma_{2}+2 e \delta\right)^{k}\left(2 e \delta \gamma_{3}+2 e \delta\right)^{l}\left(2 e \delta \gamma_{4}+2 e \delta\right)^{m} \theta_{j}^{*} \theta_{k}^{*} \theta_{l}^{*} \theta_{m}^{*}\right\}^{2} .
\end{aligned}
$$


This completes the proof of (31). In particular, $\kappa_{j, k, l, m} \in\left(N^{\otimes(j+k+l+m)}\right)^{\prime}$. It is obvious that $\widehat{\kappa}_{j, k, l, m}=\kappa_{j, k, l, m}$, namely $\kappa_{j, k, l, m} \in\left(N^{\otimes(j+k+l+m)}\right)_{s y m(j, k, l, m)}^{\prime}$.

Proof (of Theorem 11) The equality (27) is obvious from Lemma 4. We next prove identity (29). It follows from Proposition 6 that

$$
\begin{aligned}
\| \Xi_{j, k, l, m}\left(\kappa_{j, k, l, m}\right) S^{a, b}, S^{c, d} \rrbracket & =\left\langle\kappa_{j, k, l, m}, c^{\otimes j} \otimes d^{\otimes k} \otimes a^{\otimes l} \otimes b^{\otimes m}\right) e^{\langle a, c\rangle} e^{\langle b, d\rangle} \\
& =A_{j, k, l, m}(a, b, c, d) e^{\langle a, c\rangle} e^{\langle b, d\rangle} .
\end{aligned}
$$

Hence, in view of (32),

$$
\begin{aligned}
e^{-\langle a, c\rangle} e^{-\langle b, d\rangle} \sum_{j, k, l, m=0}^{\infty}\left\langle\left\langle\Xi_{j, k, l, m}\left(\kappa_{j, k, l, m}\right) S^{a, b}, S^{c, d}\right\rangle\right\rangle & =\sum_{j, k, l, m=0}^{\infty} A_{j, k, l, m}(a, b, c, d) \\
& =\psi(a, b, c, d),
\end{aligned}
$$

and therefore,

$$
\begin{aligned}
\left.\sum_{j, k, l, m=0}^{\infty}\left\langle\left\langle\Xi_{j, k, l, m}\left(\kappa_{j, k, l, m}\right) S^{a, b}, S^{c, d}\right\rangle\right\rangle\right\rangle & =e^{\langle a, c\rangle} e^{\langle b, d\rangle} \psi(a, b, c, d) \\
& =\Theta(a, b, c, d) .
\end{aligned}
$$

It follows from the uniqueness of the Taylor coefficients that $\left\{A_{j, k, l, m}\right\}_{j, k, l, m=0}^{\infty}$ is unique, and therefore so is $\left\{\kappa_{j, k, l, m}\right\}_{j, k, l, m=0}^{\infty}$ under the condition that $\widehat{\kappa}_{j, k, l, m}=\kappa_{j, k, l, m}$. We then prove that $\sum_{j, k, l, m=0}^{\infty} \Xi_{j, k, l, m}\left(\kappa_{j, k, l, m}\right) S$ converges in $\mathscr{U}_{\theta}^{*}$ for any $S \in \mathscr{U}_{\theta}$. It follows from Theorem 9 that

$$
\left\|\Xi_{j, k, l, m}\left(\kappa_{j, k, l, m}\right) S\right\|_{\theta,-\alpha-1,\left(\gamma_{1}, \gamma_{2}\right)} \leq M(j, k, l, m)\left|\kappa_{j, k, l, m}\right|_{-(\alpha+1)}\|S\|_{\theta, \alpha+1,\left(\gamma_{1}, \gamma_{2}\right)} .
$$

In view of (31) we obtain

$$
\begin{aligned}
&\left\|\Xi_{j, k, l, m}\left(\kappa_{j, k, l, m}\right) S\right\|_{\theta,-\alpha-1,\left(\gamma_{1}, \gamma_{2}\right)} \\
& \leq M(j, k, l, m) K(2 e \delta)^{j+k+l+m}\left(\gamma_{1}+1\right)^{j}\left(\gamma_{2}+1\right)^{k}\left(\gamma_{3}+1\right)^{l}\left(\gamma_{4}+1\right)^{m} \\
& \times \theta_{j}^{*} \theta_{k}^{*} \theta_{l}^{*} \theta_{m}^{*}\|S\|_{\theta, \alpha+1,\left(\gamma_{1}, \gamma_{2}\right)} \\
& \leq M_{1} K\left\{\left(8 e \delta \sqrt{\gamma_{4}}\left(\gamma_{1}+1\right)\right)^{j} j ! \theta_{j} \theta_{j}^{*}\right\}\left\{\left(8 e \delta \sqrt{\gamma_{3}}\left(\gamma_{2}+1\right)\right)^{k} k ! \theta_{k} \theta_{k}^{*}\right\} \\
& \times\left\{\left(8 e \delta \sqrt{\gamma_{1}}\left(\gamma_{3}+1\right)\right)^{l} l ! \theta_{l} \theta_{l}^{*}\right\}\left\{\left(8 e \delta \sqrt{\gamma_{2}}\left(\gamma_{4}+1\right)\right)^{m} m ! \theta_{m} \theta_{m}^{*}\right\}\|S\|_{\theta,-\alpha-1,\left(\gamma_{1}, \gamma_{2}\right) .}
\end{aligned}
$$

Since $\theta_{n} \theta_{n}^{*}=\left(\frac{e}{n}\right)^{n}$ for every $n \geq 0$, see [23], then the series

$$
\sum_{j, k, l, m=0}^{\infty}\left\|\boldsymbol{\Xi}_{j, k, l, m}\left(\kappa_{j, k, l, m}\right) S\right\|_{\theta,-\alpha-1,\left(\gamma_{1}, \gamma_{2}\right)}
$$

converges for any $S \in \mathscr{U}_{\theta}$ if we chose $\gamma_{1}, \gamma_{2}>0$ such that

$$
L\left(\gamma_{1}, \gamma_{4}\right):=8 e \delta \sqrt{\gamma_{4}}\left(\gamma_{1}+1\right)<1, \quad L\left(\gamma_{2}, \gamma_{3}\right)<1, \quad L\left(\gamma_{3}, \gamma_{1}\right)<1 \quad \text { and } L\left(\gamma_{4}, \gamma_{2}\right)<1 \text {. }
$$

Then, $\exists C \geq 0$ such that

$$
\sum_{j, k, l, m=0}^{\infty}\left\|\Xi_{j, k, l, m}\left(\kappa_{j, k, l, m}\right) S\right\|_{\theta,-\alpha-1,\left(\gamma_{1}, \gamma_{2}\right)} \leq C\|S\|_{\theta, \alpha+1,\left(\gamma_{1}, \gamma_{2}\right)} .
$$

This means that the series (29) converges in $\mathscr{U}_{\theta}^{*}$ and $\Xi Q \in \mathscr{L}\left(\mathscr{U}_{\theta}, \mathscr{U}_{\theta}^{*}\right)$. Finally we see that

$$
\begin{aligned}
\sigma^{Q}\left(\Xi^{Q}\right)(a, b, c, d) & =\| \Xi S^{a, b}, S^{c, d} \rrbracket \\
& =\sum_{j, k, l, m=0}^{\infty} \| \Xi_{j, k, l, m}\left(\kappa_{j, k, l, m}\right) S^{a, b}, S^{c, d} \rrbracket \\
& =\Theta(a, b, c, d) .
\end{aligned}
$$

For all $a, b, c, d \in N$ as desired. 
Theorem 12 For any $\Xi^{Q} \in \mathscr{L}\left(\mathscr{U}_{\theta}, \mathscr{U}_{\theta}^{*}\right)$ there exists a unique family of distributions $\kappa_{j, k, l, m}$ $\in\left(N^{\otimes(j+k+l+m)}\right)_{\text {sym }(j, k, l, m)}^{\prime}$, such that

$\Xi^{Q} S=\sum_{j, k, l, m=0}^{\infty} \Xi_{j, k, l, m}\left(\kappa_{j, k, l, m}\right) S, \quad S \in \mathscr{U}_{\theta}$,

where the righthand side converges in $\mathscr{U}_{\theta}^{*}$.

Proof For a given $\Xi^{Q} \in \mathscr{L}\left(\mathscr{U}_{\theta}, \mathscr{U}_{\theta}^{*}\right)$ we put

$\Theta(a, b, c, d)=\sigma^{Q}\left(\Xi^{Q}\right)(a, b, c, d)=\left\|\Xi^{Q} S^{a, b}, S^{c, d}\right\|, \quad a, b, c, d \in N$.

Then, by Theorem 10 we see that $\Theta \in \mathscr{G}_{\theta^{*}}\left(N^{4}\right)$. Therefore, by Theorem 11 there exists a unique family of kernels $\left\{\kappa_{j, k, l, m}\right\}_{j, k, l, m=0}^{\infty}, \kappa_{j, k, l, m} \in\left(N^{\otimes(j+k+l+m)}\right)_{s y m(j, k, l, m)}^{\prime}$ such that

$\Theta(a, b, c, d)=\sum_{j, k, l, m=0}^{\infty}\left\|\Xi_{j, k, l, m}\left(\kappa_{j, k, l, m}\right) S^{a, b}, S^{c, d}\right\|, \quad a, b, c, d \in N$.

Furthermore, as is stated in Theorem 11,

$\Xi^{\prime} S=\sum_{j, k, l, m=0}^{\infty} \Xi_{j, k, l, m}\left(\kappa_{j, k, l, m}\right) S, \quad S \in \mathscr{U}_{\theta}$,

converges in $\mathscr{U}_{\theta}^{*}, \Xi^{\prime} Q \in \mathscr{L}\left(\mathscr{U}_{\theta}, \mathscr{U}_{\theta}^{*}\right)$ and $\sigma^{Q}\left(\Xi^{\prime} Q\right)(a, b, c, d)=\Theta(a, b, c, d)$ for all $a, b, c, d \in N$. The last identity and (33) yield

$\left\|\Xi^{\prime} Q S^{a, b}, S^{c, d} \rrbracket=\right\| \Xi^{Q} S^{a, b}, S^{c, d} \rrbracket, \quad a, b, c, d \in N$.

Since $\left\{S^{a, b}, \quad a, b \in N\right\}$ span a dense subspace of $\mathscr{U}_{\theta}$ and both $\Xi^{Q}$ and $\Xi^{\prime} Q$ are continuous, we conclude that $\Xi^{Q}=\Xi^{\prime}$.

Example 1 For $z \in N^{\prime}$, the QWN-derivatives and their adjoints studied in [16] and [6] coincide respectively with

$\Xi_{0,0,1,0}(z)=D_{z}^{+}, \quad \Xi_{0,0,0,1}(z)=D_{z}^{-}$

$\Xi_{1,0,0,0}(z)=\left(D_{z}^{+}\right)^{*}, \quad \Xi_{0,1,0,0}(z)=\left(D_{z}^{-}\right)^{*}$

Example 2 For $K_{1}, K_{2} \in \mathscr{L}\left(N^{\prime}, N^{\prime}\right)$, the QWN-Laplacians studied in [6] (see also [15]) and their adjoints are given by

$$
\begin{aligned}
\Delta_{G}^{Q}\left(K_{1}, K_{2}\right) & =\int_{\mathbb{R}^{2}} \tau_{K_{1}}(s, t) D_{s}^{+} D_{t}^{+} \mathrm{d} s \mathrm{~d} t+\int_{\mathbb{R}^{2}} \tau_{K_{2}}(s, t) D_{s}^{-} D_{t}^{-} \mathrm{d} s \mathrm{~d} t \\
& =\Xi_{0,0,2,0}\left(\tau_{K_{1}}\right)+\Xi_{0,0,0,2}\left(\tau_{K_{2}}\right) \\
N_{K_{1}, K_{2}}^{Q} & =\int_{\mathbb{R}^{2}} \tau_{K_{1}}(s, t)\left(D_{s}^{+}\right)^{*} D_{s}^{+} \mathrm{d} s \mathrm{~d} t+\int_{\mathbb{R}^{2}} \tau_{K_{2}}(s, t)\left(D_{s}^{-}\right)^{*} D_{t}^{-} \mathrm{d} s \mathrm{~d} t \\
& =\Xi_{1,0,1,0}\left(\tau_{K_{1}}\right)+\Xi_{0,1,0,1}\left(\tau_{K_{2}}\right) \\
\left(\Delta_{G}^{Q}\right)^{*}\left(K_{1}, K_{2}\right) & =\int_{\mathbb{R}^{2}} \tau_{K_{1}}(s, t)\left(D_{s}^{+}\right)^{*}\left(D_{t}^{+}\right)^{*} \mathrm{~d} s \mathrm{~d} t+\int_{\mathbb{R}^{2}} \tau_{K_{2}}(s, t)\left(D_{s}^{-}\right)^{*}\left(D_{t}^{-}\right)^{*} \mathrm{~d} s \mathrm{~d} t \\
& =\Xi_{2,0,0,0}\left(\tau_{K_{1}}\right)+\Xi_{0,2,0,0}\left(\tau_{K_{2}}\right) .
\end{aligned}
$$


Example 3 For $K_{1}, K_{2}, B_{1}, B_{2} \in \mathscr{L}\left(N^{\prime}, N^{\prime}\right)$, the QWN-Fourier Gauss transform studied in [15] and [6] is given by

$$
\begin{aligned}
G_{K_{1}, K_{2} ; B_{1}, B_{2}}^{Q}= & \sum_{j, k, l, m=0}^{\infty} \frac{1}{j ! k ! l ! m !} \int_{\mathbb{R}^{2(j+k+l+m)}} \kappa\left(s_{1}, \cdots, s_{j}, t_{1}, \cdots, t_{k}, u_{1}, \cdots, u_{2 l+j}, v_{1}, \cdots, v_{2 m+k}\right) \\
& \left(D_{s_{1}}^{+}\right)^{*} \cdots\left(D_{s_{j}}^{+}\right)^{*}\left(D_{t_{1}}^{-}\right)^{*} \cdots\left(D_{t_{k}}^{-}\right)^{*} D_{u_{1}}^{+} \cdots D_{u_{j+2 l}}^{+} D_{v_{1}}^{-} \cdots D_{v_{k+2 m}}^{-} \\
& \mathrm{d} s_{1} \cdots \mathrm{d} s_{j} \mathrm{~d} t_{1} \cdots \mathrm{d} t_{k} \mathrm{~d} u_{1} \cdots \mathrm{d} u_{j+2 l} \mathrm{~d} v_{1} \cdots \mathrm{d} v_{k+2 m} \\
= & \sum_{j, k, l, m=0}^{\infty} \frac{1}{j ! k ! l ! m !} \Xi_{j, k, 2 l+j, 2 m+k}(\kappa),
\end{aligned}
$$

where $\kappa$ is given by

$$
\begin{aligned}
& \kappa\left(s_{1}, \cdots, s_{j}, t_{1}, \cdots, t_{k}, u_{1}, \cdots, u_{2 l+j}, v_{1}, \cdots, v_{2 m+k}\right) \\
& =\tau_{K_{1}}\left(u_{1}, u_{2}\right) \cdots \tau_{K_{1}}\left(u_{2 l-1}, u_{2 l}\right) \tau_{K_{2}}\left(v_{1}, v_{2}\right) \cdots \tau_{K_{2}}\left(v_{2 m-1}, v_{2 m}\right) \\
& \quad \times \tau_{\left(B_{1}-I\right)}\left(s_{1}, u_{2 l+1}\right) \cdots \tau_{\left(B_{1}-I\right)}\left(s_{j}, u_{2 l+j}\right) \tau_{\left(B_{2}-I\right)}\left(t_{1}, v_{2 m+1}\right) \cdots \tau_{\left(B_{2}-I\right)}\left(t_{k}, v_{2 m+k}\right) .
\end{aligned}
$$

Example 4 For $c, d \in N$, the QWN-translation operator admits the following representation (see [5])

$$
\begin{aligned}
& T_{-c,-d}^{Q} \\
& \quad=\sum_{l, m=0}^{\infty} \frac{1}{l ! m !} \int_{\mathbb{R}^{l+m}} \mathrm{~d}\left(s_{1}\right) \cdots \mathrm{d}\left(s_{l}\right) c\left(t_{1}\right) \cdots c\left(t_{m}\right) D_{s_{1}}^{+} \cdots D_{s_{l}}^{+} D_{t_{1}}^{-} \cdots D_{t_{m}}^{-} \mathrm{d} s_{1} \cdots \mathrm{d} s_{l} \mathrm{~d} t_{1} \cdots \mathrm{d} t_{m} \\
& =\sum_{l, m=0}^{\infty} \frac{1}{l ! m !} \Xi_{0,0, l, m}\left(d^{\otimes l} \otimes c^{\otimes m}\right) .
\end{aligned}
$$

Example 5 Let $S=\sum_{i, j=0}^{\infty} \Xi_{i, j}\left(s_{i, j}\right) \in \mathscr{U}_{\theta}^{*}$. Then, the QWN-convolution operator $C_{S}^{Q}$, defined in [5], coincides with

$$
\begin{aligned}
& C_{S}^{Q}=\sum_{i, j=0}^{\infty} \int_{\mathbb{R}^{i+j}} s_{i, j}\left(u_{1}, \cdots, u_{i}, v_{1}, \cdots, v_{j}\right) D_{u_{1}}^{+} \cdots D_{u_{i}}^{+} D_{v_{1}}^{-} \cdots D_{v_{j}}^{-} \mathrm{d} u_{1} \cdots \mathrm{d} u_{i} \mathrm{~d} v_{1} \cdots \mathrm{d} v_{j} \\
& =\sum_{i, j=0}^{\infty} \Xi_{0,0, i, j}\left(s_{i, j}\right) .
\end{aligned}
$$

\section{QWN-rotation group}

Let $O(X, H)$ given by (see [21])

$O(X, H)=\left\{B \in G L(X) ;|B \xi|_{0}=|\xi|_{0} \forall \xi \in X\right\}$,

which is called infinite-dimensional rotation group. We say that a continuous operator from $\mathscr{U}_{\theta}$ into $\mathscr{U}_{\theta}^{*}$ is rotationinvariant if

$\left(\Gamma^{Q}(B)\right)^{*} \Xi^{Q} \Gamma^{Q}(B)=\Xi^{Q}, \quad \forall B \in O(X, H)$,

where $\Gamma^{Q}(B)$ is given by

$\Gamma^{Q}(B) \Xi=\sum_{l, m} \Xi_{l, m}\left(B^{\otimes(l+m)} \kappa_{l, m}\right)$,

for $\Xi=\sum_{l, m} \Xi_{l, m}\left(\kappa_{l, m}\right)$ in $\mathscr{U}_{\theta}$. By definition, if $\Xi^{Q}$ is rotation invariant, so is $\left(\Xi^{Q}\right)^{*}$. 
The main purpose of this section is to characterize all rotation-invariant operators $\Xi^{Q}$ in terms of the QWN-Gross Laplacian and the QWN-conservation operator given by

$$
\begin{aligned}
& \Delta_{G}^{Q}=\int_{\mathbb{R}^{2}} \tau(s, t) D_{s}^{+} D_{t}^{+} \mathrm{d} s \mathrm{~d} t+\int_{\mathbb{R}^{2}} \tau(s, t) D_{s}^{-} D_{t}^{-} \mathrm{d} s \mathrm{~d} t \\
& N^{Q}=\int_{\mathbb{R}^{2}} \tau(s, t)\left(D_{s}^{+}\right)^{*} D_{s}^{+} \mathrm{d} s \mathrm{~d} t+\int_{\mathbb{R}^{2}} \tau(s, t)\left(D_{s}^{-}\right)^{*} D_{t}^{-} \mathrm{d} s \mathrm{~d} t .
\end{aligned}
$$

We recall that (see [21]) $F \in\left(N^{\otimes n}\right)^{\prime}$ is rotation-invariant if $\left(B^{\otimes n}\right)^{*} F=F$ for all $B \in O(X, H)$.

Theorem 13 Let $\Xi^{Q} \in \mathscr{L}\left(\mathscr{U}_{\theta}, \mathscr{U}_{\theta}^{*}\right)$ and $\Xi^{Q}=\sum_{j, k, l, m=0}^{\infty} \Xi_{j, k, l, m}(\kappa)$, where $\kappa \in\left(N^{\otimes(j+k+l+m)}\right)_{\text {sym }(j, k, l, m)}^{\prime}$. Then $\Xi^{Q}$ is rotation invariant if and only if $\kappa$ is rotation invariant.

Proof Let $\Xi^{Q} \in \mathscr{L}\left(\mathscr{U}_{\theta}, \mathscr{U}_{\theta}^{*}\right)$ given by $\Xi^{Q}=\sum_{j, k, l, m=0}^{\infty} \Xi_{j, k, l, m}(\kappa)$, where $\kappa \in\left(N^{\otimes(j+b+l+m)}\right)_{s y m(j, k, l, m)}^{\prime}$. Recall that $\Gamma^{Q}(B) \Xi^{a, b}=\Xi^{B a, B b}, a, b \in N$. Then, for any $a, b \in N$ we have

$$
\begin{aligned}
\sigma^{Q}\left(\left(\Gamma^{Q}(B)\right)^{*} \Xi^{Q} \Gamma^{Q}(B)\right)(a, b, c, d) & =\|\left(\Gamma^{Q}(B)\right)^{*} \Xi^{Q} \Gamma^{Q}(B) \Xi^{a, b}, \Xi^{c, d} \rrbracket \\
& =\| \Xi^{Q} \Gamma^{Q}(B) \Xi^{a, b}, \Gamma_{(B)}^{Q} \Xi^{c, d} \rrbracket \\
& =\| \Xi^{Q} \Xi^{B a, B b}, \Xi^{B c, B d} \rrbracket \\
& =\sigma^{Q}\left(\Xi^{Q}\right)(B a, B b, B c, B d) .
\end{aligned}
$$

From Proposition 6, we obtain

$$
\begin{aligned}
& \left.\sigma\left(\left(\Gamma^{Q}(B)\right)^{*} \Xi^{Q} \Gamma^{Q}\right)\right)(a, b, c, d) \\
& =e^{\langle B a, B c\rangle+\langle B b, B d\rangle} \sum_{j, k, l, m=0}^{\infty}\left\langle\kappa,(B c)^{\otimes j} \otimes(B d)^{\otimes k} \otimes(B a)^{\otimes l} \otimes(B b)^{\otimes m}\right\rangle .
\end{aligned}
$$

Then, using the obvious equality, for $\Xi^{Q}=\sum_{j, k, l, m} \Xi_{j, k, l, m}(\kappa)$

$e^{-\langle a, c\rangle-\langle b, d\rangle} \sigma^{Q}\left(\Xi^{Q}\right)(a, b, c, d)=\sum_{j, k, l, m}^{\infty}\left\langle\kappa, c^{\otimes j} \otimes d^{\otimes k} \otimes a^{\otimes l} \otimes b^{\otimes m}\right\rangle$.

We get

$$
\begin{aligned}
& \sigma\left(\left(\Gamma^{Q}(B)\right)^{*} \Xi^{Q} \Gamma^{Q}(B)\right)(a, b, c, d) \\
= & e^{\langle a, c\rangle+\langle b, d\rangle} \sum_{j, k, l, m=0}^{\infty}\left\langle\left(B^{\otimes(j+k+l+m)}\right)^{*} \kappa, c^{\otimes j} \otimes d^{\otimes k} \otimes a^{\otimes l} \otimes b^{\otimes m}\right\rangle \\
= & \sigma^{Q}\left(\sum_{j, k, l, m=0}^{\infty} \Xi_{j, k, l, m}\left(\left(B^{\otimes(j+k+l+m)}\right)^{*} \kappa\right)\right)(a, b, c, d) .
\end{aligned}
$$

In particular, $\left(\Gamma^{Q}(B)\right)^{*} \Xi_{j, k, l, m}(x) P^{Q}(B)=\Xi_{j, k, l, m}\left(\left(B^{\otimes(j+k+l+m)}\right)^{*} \kappa\right)$. Therefore, by the uniqueness of the Fock expansion, $\Xi^{Q}$ is rotation invariant if and only if $\Xi_{j, k, l, m}(\kappa)$ is rotation invariant if and only if $\kappa$ is rotation invariant.

Let $\Delta_{G}^{Q_{+}}$and $\Delta_{G}^{Q_{-}}$given by

$\Delta_{G}^{Q_{+}}=\int_{\mathbb{R}^{2}} \tau(s, t) D_{s}^{+} D_{t}^{+} \mathrm{d} s \mathrm{~d} t, \quad \Delta_{G}^{Q_{-}}=\int_{\mathbb{R}^{2}} \tau(s, t) D_{s}^{-} D_{t}^{-} \mathrm{d} s \mathrm{~d} t$. 
Lemma 5 Let $\alpha^{\prime}, \alpha^{\prime \prime}, \beta^{\prime}, \beta^{\prime \prime}, \lambda^{\prime}, \lambda^{\prime \prime}$ be non-negative integers and put $j=2 \alpha^{\prime}+\beta^{\prime}, k=2 \alpha^{\prime \prime}+\beta^{\prime \prime}, l=2 \lambda^{\prime}+\beta^{\prime}$ and $m=2 \lambda^{\prime \prime}+\beta^{\prime \prime}$. Then,

$$
\begin{aligned}
& \Xi_{j, k, l, m}\left(\tau^{\otimes \alpha^{\prime}} \otimes \tau_{\beta^{\prime}} \otimes \tau^{\otimes \lambda^{\prime}} \otimes \tau^{\otimes \alpha^{\prime \prime}} \otimes \tau_{\beta^{\prime \prime}} \otimes \tau^{\otimes \lambda^{\prime \prime}}\right) \\
& =\left(\left(\Delta_{G}^{Q_{+}}\right)^{*}\right)^{\alpha^{\prime \prime}}\left(\left(\Delta_{G}^{Q-}\right)^{*}\right)^{\alpha^{\prime}} \Xi_{\beta^{\prime}, \beta^{\prime \prime}, \beta^{\prime}, \beta^{\prime \prime}}\left(\tau_{\beta^{\prime}} \otimes \tau_{\beta^{\prime \prime}}\right)\left(\Delta_{G}^{Q^{+}}\right)^{\lambda^{\prime}}\left(\Delta_{G}^{Q^{-}}\right)^{\lambda^{\prime \prime}}
\end{aligned}
$$

where $\tau_{\beta^{\prime}} \in\left(X^{\otimes 2 \beta^{\prime}}\right)^{\prime}$ is given by

$\tau_{\beta^{\prime}}=\sum e_{j_{1}} \otimes \cdots \otimes e_{j_{\beta^{\prime}}} \otimes e_{j_{1}} \otimes \cdots \otimes e_{j_{\beta^{\prime}}}$.

Proof Since both sides of (36) are continuous operators from $\mathscr{U}_{\theta}$ into $\mathscr{U}_{\theta}^{*}$, it suffices to check that they coincide on the operator $\Xi^{a, b}$, for $a, b \in N$, or by applying the QWN-symbol map. To this end, let a,b,c,d $\in N$, then since

$\Delta_{G}^{Q^{+}} \Xi^{a, b}=\langle a, a\rangle \Xi^{a, b}, \quad \Delta_{G}^{Q^{-}} \Xi^{a, b}=\langle b, b\rangle \Xi^{a, b}$

we get,

$$
\begin{aligned}
& \sigma^{Q}\left\{\left(\left(\Delta_{G}^{Q_{+}}\right)^{*}\right)^{\alpha^{\prime \prime}}\left(\left(\Delta_{G}^{Q-}\right)^{*}\right)^{\alpha^{\prime}} \Xi_{\beta^{\prime}, \beta^{\prime \prime}, \beta^{\prime}, \beta^{\prime \prime}}\left(\tau_{\beta^{\prime}} \otimes \tau_{\beta^{\prime \prime}}\right)\left(\Delta_{G}^{Q^{+}}\right)^{\lambda^{\prime}}\left(\Delta_{G}^{Q^{-}}\right)^{\lambda^{\prime \prime}}\right\}(a, b, c, d) \\
& \left.=\| \Xi_{\beta^{\prime}, \beta^{\prime \prime}, \beta^{\prime}, \beta^{\prime \prime}}\left(\tau_{\beta^{\prime}} \otimes \tau_{\beta^{\prime \prime}}\right)\left(\Delta_{G}^{Q^{+}}\right)^{\lambda^{\prime}}\left(\Delta_{G}^{Q^{-}}\right)^{\lambda^{\prime \prime}} \Xi^{a, b},\left(\Delta_{G}^{Q^{-}}\right)^{\alpha^{\prime \prime}}\left(\Delta_{G}^{Q^{+}}\right)^{\alpha^{\prime}} \Xi^{c, d}\right\rangle \\
& =\langle a, a\rangle^{\lambda^{\prime}}\langle b, b\rangle^{\lambda^{\prime \prime}}\langle c, c\rangle^{\alpha^{\prime}}\langle d, d\rangle^{\alpha^{\prime \prime}}\left\langle\left\langle\left\langle\Xi_{\beta^{\prime}, \beta^{\prime \prime}, \beta^{\prime}, \beta^{\prime \prime}}\left(\tau_{\beta^{\prime}} \otimes \tau_{\beta^{\prime \prime}}\right) \Xi^{a, b}, \Xi^{c, d}\right\rangle\right\rangle\right\rangle \\
& =\langle a, a\rangle^{\lambda^{\prime}}\langle b, b\rangle^{\lambda^{\prime \prime}}\langle c, c\rangle^{\alpha^{\prime}}\langle d, d\rangle^{\alpha^{\prime \prime}}\left\langle\tau_{\beta^{\prime}} \otimes \tau_{\beta^{\prime \prime}}, c^{\otimes \beta^{\prime}} \otimes a^{\otimes \beta^{\prime}} \otimes d^{\otimes \beta^{\prime \prime}} \otimes b^{\otimes \beta^{\prime \prime}}\right\rangle e^{\langle a, c\rangle+\langle b, d\rangle} \\
& =\langle a, a\rangle^{\lambda^{\prime}}\langle b, b\rangle^{\lambda^{\prime \prime}}\langle c, c\rangle^{\alpha^{\prime}}\langle d, d\rangle^{\alpha^{\prime \prime}}\langle a, c\rangle^{\beta^{\prime}}\langle b, d\rangle^{\beta^{\prime \prime}} e^{\langle a, c\rangle+\langle b, d\rangle} .
\end{aligned}
$$

On the other hand, from the commutation between $\left(D_{s}^{-}\right)^{*}$ and $D_{t}^{+}$we have

$$
\begin{aligned}
& \sigma^{Q}\left(\Xi_{j, k, l, m}\left(\tau^{\otimes \alpha^{\prime}} \otimes \tau_{\beta^{\prime}} \otimes \tau^{\otimes \lambda^{\prime}} \otimes \tau^{\otimes \alpha^{\prime \prime}} \otimes \tau_{\beta^{\prime \prime}} \otimes \tau^{\otimes \lambda^{\prime \prime}}\right)\right)(a, b, c, d) \\
& =\left\langle\tau^{\otimes \alpha^{\prime}} \otimes \tau_{\beta^{\prime}} \otimes \tau^{\otimes \lambda^{\prime}} \otimes \tau^{\otimes \alpha^{\prime \prime}} \otimes \tau_{\beta^{\prime \prime}} \otimes \tau^{\otimes \lambda^{\prime \prime}}, c^{\otimes 2 \alpha^{\prime}} \otimes c^{\otimes \beta^{\prime}} \otimes a^{\otimes \beta^{\prime}} \otimes a^{\otimes 2 \lambda^{\prime}} \otimes\right. \\
& \quad d^{\otimes 2 \alpha^{\prime \prime}} \otimes d^{\otimes \beta^{\prime \prime}} \otimes b^{\otimes \beta^{\prime \prime}} \otimes b^{\otimes 2 \lambda^{\prime \prime}} \mid e^{\langle a, c\rangle+\langle b, d\rangle} \\
& =\langle c, c\rangle^{\alpha^{\prime}}\langle a, c\rangle^{\beta^{\prime}}\langle a, a\rangle^{\lambda^{\prime}}\langle d, d\rangle^{\alpha^{\prime \prime}}\langle b, d\rangle^{\beta^{\prime \prime}}\langle b, b\rangle^{\lambda^{\prime \prime}} e^{\langle a, c\rangle+\langle b, d\rangle} .
\end{aligned}
$$

Hence we complete the proof.

Theorem 14 Let $\kappa \in\left(N^{\otimes(j+k+l+m)}\right)^{\prime}$ and assume that $\Xi_{j, k, l, m}(\kappa)=0$ is rotation invariant. If $j+k+l+m$ is odd, then $\Xi_{j, k, l, m}(\kappa)=0$. If $j+k+l+m$ is even, then $\Xi_{j, k, l, m}(\kappa)$ is a linear combination of $\left(\left(\Delta_{G}^{Q}\right)^{\alpha}\right)^{*}\left(N^{Q}\right)^{\beta}\left(\Delta_{G}^{Q}\right)^{\lambda}$ with $\alpha, \beta$, $\lambda$ being non-negative integers such that $\alpha+\beta+\lambda \leq(j+k+l+m) / 2$.

Proof Suppose that $\Xi_{j, k, l, m}(\kappa)$ is rotation invariant. Without loss of generality we may assume that $\kappa \in$ $\left(N^{\otimes(j+k+l+m)}\right)_{\text {sym }(j, k, l, m)}^{\prime}$. Then, $\kappa$ is rotation invariant by Theorem 13. It is well known (see [21]) that if $F \in\left(N^{\otimes n}\right)^{\prime}$ is rotation invariant and $\mathrm{n}$ is odd then $F=0$ and if $n=2 p$ then $\mathrm{F}$ is a linear combination of $\left(\tau^{\otimes p}\right)^{\sigma}$ for $\sigma \in G_{n}$. From which we deduce that if $j+k+l+m$ is odd then $\kappa=0$ and hence $\Xi_{j, k, l, m}(\kappa)=0$.

We consider now the case when $j+k+l+m$ is even. Then $\kappa$ is a linear combination of $\left(\tau^{\otimes(j+k+l+m) / 2}\right)^{\sigma}$, $\sigma \in G_{j+k+l+m}$. For each $\sigma \in G_{j+k+l+m}$ we may find $\sigma^{\prime} \in G_{j} \times G_{k} \times G_{l} \times G_{m}$ such that

$$
\begin{aligned}
\left(\tau^{\otimes(j+k+l+m) / 2}\right)^{\sigma \sigma^{\prime}}= & \sum e_{i_{1}}^{\otimes 2} \otimes \cdots \otimes e_{i_{\alpha^{\prime}}}^{\otimes 2} \otimes e_{j_{1}}^{\otimes 2} \otimes \cdots \otimes e_{j_{\beta^{\prime}}}^{\otimes 2} \otimes e_{k_{1}}^{\otimes 2} \otimes \cdots \otimes e_{k_{\lambda^{\prime}}}^{\otimes 2} \otimes \\
& e_{n_{1}}^{\otimes 2} \otimes \cdots \otimes e_{n_{\alpha^{\prime \prime}}}^{\otimes 2} \otimes e_{l_{1}}^{\otimes 2} \otimes \cdots \otimes e_{\beta_{\beta^{\prime \prime}}}^{\otimes 2} \otimes e_{m_{1}}^{\otimes 2} \otimes \cdots \otimes e_{m_{\lambda^{\prime \prime}}}^{\otimes 2} \\
= & \tau^{\otimes \alpha^{\prime}} \otimes \tau_{\beta^{\prime}} \otimes \tau^{\otimes \lambda^{\prime}} \otimes \tau^{\otimes \alpha^{\prime \prime}} \otimes \tau_{\beta^{\prime \prime}} \otimes \tau^{\otimes \lambda^{\prime \prime}}
\end{aligned}
$$


for some non-negative integers $\alpha^{\prime}, \alpha^{\prime \prime}, \beta^{\prime}, \beta^{\prime \prime}, \lambda^{\prime}, \lambda^{\prime \prime}$ with $j=2 \alpha^{\prime}+\beta^{\prime}, k=2 \alpha^{\prime \prime}+\beta^{\prime \prime}, l=2 \lambda^{\prime}+\beta^{\prime}$ and $m=2 \lambda^{\prime \prime}+\beta^{\prime \prime}$. Then in view of Lemma 5 , we have

$$
\begin{aligned}
& \Xi_{j, k, l, m}\left(\left(\tau^{\otimes(j+k+l+m) / 2}\right)^{\sigma}\right) \\
= & \Xi_{j, k, l, m}\left(\left(\tau^{\otimes(j+k+l+m) / 2}\right)^{\sigma \sigma^{\prime}}\right) \\
= & \left(\left(\Delta_{G}^{Q_{+}}\right)^{\alpha^{\prime \prime}}\right)^{*}\left(\left(\Delta_{G}^{Q_{-}}\right)^{\alpha^{\prime}}\right)^{*} \Xi_{\beta^{\prime}, \beta^{\prime \prime}, \beta^{\prime}, \beta^{\prime \prime}}\left(\tau_{\beta^{\prime}} \otimes \tau_{\beta^{\prime \prime}}\right)\left(\Delta_{G}^{Q_{+}}\right)^{\lambda^{\prime}}\left(\Delta_{G}^{Q_{-}}\right)^{\lambda^{\prime \prime}} .
\end{aligned}
$$

On the other hand

$$
\begin{aligned}
\sum_{\alpha^{\prime}=0}^{\alpha} \frac{\alpha !}{\alpha !\left(\alpha-\alpha^{\prime}\right) !}\left(\Delta_{G}^{Q_{+}}\right)^{\alpha^{\prime}}\left(\Delta_{G}^{Q_{-}}\right)^{\alpha-\alpha^{\prime}} \Xi^{c, d} & =\sum_{\alpha^{\prime}=0}^{\alpha} \frac{\alpha !}{\alpha !\left(\alpha-\alpha^{\prime}\right) !}\langle c, c\rangle^{\alpha^{\prime}}\langle d, d\rangle^{\alpha-\alpha^{\prime}} \Xi^{c, d} \\
& =(\langle c, c\rangle+\langle d, d\rangle)^{\alpha} \Xi^{c, d} \\
& =\left(\Delta_{G}^{Q}\right)^{\alpha} \Xi^{c, d}
\end{aligned}
$$

and similarly,

$$
\sum_{\lambda^{\prime}=0}^{\lambda} \frac{\lambda !}{\lambda !\left(\lambda-\lambda^{\prime}\right) !}\left(\Delta_{G}^{Q_{+}}\right)^{\lambda^{\prime}}\left(\Delta_{G}^{Q_{-}}\right)^{\lambda-\lambda^{\prime}} \Xi^{a, b}=\left(\Delta_{G}^{Q}\right)^{\lambda} \Xi^{a, b} .
$$

Then it suffices to prove that $\Xi_{\beta^{\prime}, \beta^{\prime \prime}, \beta^{\prime}, \beta^{\prime \prime}}\left(\tau_{\beta^{\prime}} \otimes \tau_{\beta^{\prime \prime}}\right)$ is a polynomial in the QWN-conservation operator $N^{Q}$ or in $N^{Q_{+}}$and $N^{Q_{-}}$, where $N^{Q}=N^{Q_{-}}+N^{Q_{+}}$, in other words it suffices to prove that

$\Xi_{\beta^{\prime}, \beta^{\prime \prime}, \beta^{\prime}, \beta^{\prime \prime}}\left(\tau_{\beta^{\prime}} \otimes \tau_{\beta^{\prime \prime}}\right)=N^{Q_{+}}\left(N^{Q_{+}}-1\right) \cdots\left(N^{Q_{+}}-\beta^{\prime}+1\right) N^{Q_{-}}\left(N^{Q_{-}}-1\right) \cdots\left(N^{Q_{-}}-\beta^{\prime \prime}+1\right)$.

To this end, denote by $\mathrm{P}$ the righthand side of (37), then

$$
\begin{aligned}
\sigma^{Q}(P)(a, b, c, d)= & \sum_{l=\beta^{\prime}, m=\beta^{\prime \prime}}^{\infty} l(l-1) \cdots\left(l-\beta^{\prime}+1\right) m(m-1) \cdots\left(m-\beta^{\prime \prime}+1\right) \\
& \times\left\langle\frac{a^{\otimes l}}{l !} \otimes \frac{b^{\otimes m}}{m !}, c^{\otimes l} \otimes d^{\otimes m}\right\rangle \\
= & \langle a, c\rangle^{\beta^{\prime}}\langle b, d\rangle^{\beta^{\prime \prime}} e^{\langle a, c\rangle+\langle b, d\rangle} \\
= & \sigma^{Q}\left(\Xi_{\beta^{\prime}, \beta^{\prime \prime}, \beta^{\prime}, \beta^{\prime \prime}}\left(\tau_{\beta^{\prime}} \otimes \tau_{\beta^{\prime \prime}}\right)\right)(a, b, c, d) .
\end{aligned}
$$

Hence we complete the proof.

\section{References}

1. Accardi, L., Barhoumi, A., Ji, U.C.: Quantum Laplacians on generalized operators on Boson Fock space. Probab. Math. Stat. 31, 1-24 (2011)

2. Accardi, L., Ouerdiane, H., Smolyanov, O.G.: Lévy Laplacian acting on operators. Russ. J. Math. Phys. 10(4), 359-380 (2003)

3. Accardi, L., Smolyanov, O.G.: On Laplacians and Traces. Conferenze del Seminario di Matematica dell’Università di Bari 250, $1-28(1993)$

4. Accardi, L., Smolyanov, O.G.: Transformations of Gaussian measures generated by the Lévy Laplacian and generalized traces. Dokl. Akad. Nauk SSSR 350, 5-8 (1996)

5. Barhoumi, A., Lanconelli, A., Rguigui, H.: QWN-convolution operators with application to differential equations. Random Oper. Stoch. Equ. 22(4), 195-211 (2014)

6. Barhoumi, A., Ouerdiane, H., Rguigui, H.: QWN-Euler operator and associated cauchy problem. Infinite Dimens. Anal. Quantum Probab. Related Topics 15(1), 1250004 (20 pages) (2012)

7. Barhoumi, A., Ouerdiane, H., Rguigui, H.: Stochastic heat equation on algebra of generalized functions. Infinite Dimens. Anal. Quantum Probab. Related Topics 15(4), 1250026 (18 pages) (2012)

8. Chung, D.M., Ji, U.C.: Transform on white noise functionals with their application to Cauchy problems. Nagoya Math. J. 147, 1-23 (1997) 
9. Chung, D.M., Ji, U.C.: Transformation groups on white noise functionals and their application. Appl. Math. Optim. 37(2), 205-223 (1998)

10. Gannoun, R., Hachaichi, R., Ouerdiane, H., Rezgi, A.: Un théorème de dualité entre espace de fonction holomorphes à croissance exponentielle. J. Funct. Anal. 171, 1-14 (2000)

11. Gel'fand, I.M., Shilov, G.E.: Generalized functions, vol. I. Academic Press Inc, New York (1968)

12. Gross, L.: Abstract Wiener spaces. Proc. 5-th Berkeley Symp. Math. Stat. Probab. 2, 31-42 (1967)

13. Hida, T.: A role of the Lévy Laplacian in the causal calculus of generalized white noise functionals. In: Cambanis S., (ed.) Stoch. Proc., A Festschrift in Honour of G. Kallianpur. Springer (1992)

14. Hida, T., Obata, N., Saitô, K.: Infinite dimensional rotation and Laplacians in terms of white noise calculus. Nagoya Math. J. 128, 65-93 (1992)

15. Ji, U.C.: Quantum extensions of Fourier-Gauss and Fourier-Mehler transforms. J. Korean Math. Soc. 45(6), 1785-1801 (2008)

16. Ji, U.C., Obata, N.: Annihilation-derivative, creation-derivative and representation of quantum martingales. Commun. Math. Phys. 286, 751-775 (2009)

17. Ji, U.C., Obata, N., Ouerdiane, H.: Analytic characterization of generalized Fock space operators as two-variable entire function with growth condition. Infinite Dimens. Anal. Quantum Probab. Related Topics 5(3), 395-407 (2002)

18. Khalgui, M.S., Torasso, P.: Poisson-Plancherel formula for a real semialgebraic group. 1. Fourier-Transform of orbital integrals. J. Funct. Anal. 116(2), 359-440 (1993)

19. Kuo, H.H.: White noise distribution theory. CRC Press, Boca Raton (1996)

20. Lee, Y.J.: Integral transform of analytic function on abstract Wiener space. J. Funct. Anal. 47(2), 153-164 (1982)

21. Obata, N.: White noise calculus and Fock spaces. Lecture notes in Mathematics, vol. 1577. Spriger (1994)

22. Obata, N.: Derivations on white noise functionals. Nagoya Math. J. 139, 21-36 (1995)

23. Obata, N.: Quantum white noise calculus based on nuclear algebras of entire function. Trends Infinite Dimens. Anal. Quantum Probab (Kyoto 2001) RIMS 1278, 130-157 (2002)

24. Ouerdiane, H., Rguigui, H.: QWN-conservation operator and associated wick differential equation. Commun. Stoch. Anal. 6(3), 437-450 (2012)

25. Parthasarathy, K.R.: An introduction to quantum stochastic calculus. Birkhäuser (1992)

26. Piech, M.A.: Parabolic equations associated with the number operator. Trans. Am. Math. Soc. 194, $213-222$ (1974)

27. Potthoff, J., Yan, J.A.: Some results about test and generalized functionals of white noise. In: Chen et al. L.Y. (ed.) Proc. Singapore Prob. Conf., pp. 121-145 (1989)

28. Rguigui, H.: Quantum Ornstein-Uhlenbeck semigroups. Quantum Studies: Mathematics and Foundations. doi:10.1007/ s40509-014-0023-5

29. Rguigui, H.: Quantum $\lambda$-potentials associated to quantum Ornstein-Uhlenbeck semigroups. Chaos Solitons Fractals 73, 80-89 (2015) 